\title{
Dichotomies pour les espaces de suites réelles
}

\author{
par
}

\author{
Pierre Casevitz (Caen)
}

\begin{abstract}
There is a general conjecture, the dichotomy (C) about Borel equivalence relations $E$ : (i) $E$ is Borel reducible to the equivalence relation $E_{G}^{X}$ where $X$ is a Polish space, and $G$ a Polish group acting continuously on $X$; or (ii) a canonical relation $E_{1}$ is Borel reducible to $E$. (C) is only proved for special cases as in [So].

In this paper we make a contribution to the study of $(\mathrm{C})$ : a stronger conjecture is true for hereditary subspaces of the Polish space $\mathbb{R}^{\omega}$ of real sequences, i.e., subspaces such that $\left[y=\left(y_{n}\right)_{n} \in X\right.$ and $\left.\forall n,\left|x_{n}\right| \leq\left|y_{n}\right|\right] \Rightarrow x=\left(x_{n}\right)_{n} \in X$. If such an $X$ is analytic as a subset of $\mathbb{R}^{\omega}$, then either $X$ is Polishable as a vector subspace, or $X$ admits a subspace strongly isomorphic to the space $c_{00}$ of finite sequences, or to the space $\ell_{\infty}$ of bounded sequences.

When $X$ is Polishable, the metrics have a very simple form as in the case studied in [So], which allows us to study precisely the properties of those $X$ 's.
\end{abstract}

Il s'agit ici d'étudier dans le contexte des sous-espaces de l'espace polonais des suites de réels une conjecture qui était déjà connue pour d'autres types de groupes, sans structure vectorielle :

Si $E$ est une relation d'équivalence analytique sur un espace borélien standard $Z$, on est dans un des deux cas suivants :

- ou bien $E$ se plonge dans une relation $E_{G}^{X}$ induite par une action polonaise sur un espace polonais $X$,

- ou bien $E_{1}$ se plonge dans $E$, où $E_{1}$ est l'égalité dans $2^{\omega^{2}}$ modulo l'idéal $I_{1}$ des parties de $\omega^{2}$ contenues dans un nombre fini de verticales $\{n\} \times \omega$.

(Ici une relation $E$ sur $X$ se plonge dans une relation d'équivalence $F$ sur $Y$ s'il y a une fonction $f: X \rightarrow Y$ telle que $f(x) F f\left(x^{\prime}\right) \Leftrightarrow x E x^{\prime}$, et les plongements sont exigés boréliens.)

2000 Mathematics Subject Classification: Primary 03E15, 06F20, 46A45, 54D55, 54H05; Secondary 06F30, 46B40, 54E52, 54D65.

Key words and phrases: Borel complexity, subspaces of real sequences, topology of subspaces of real sequences, Polishable spaces, dichotomy theorems, Borel equivalence relations. 
La justification de cette conjecture est qu'on ne peut pas plonger $E_{1}$ dans une relation induite par une action polonaise [K-L]; donc, étant donnée une relation $E$, on ne peut à la fois plonger $E$ dans une $E_{G}^{X}$ et voir $E_{1}$ se plonger dans $E$. Les exemples connus ne font pas apparaître pour l'instant de statut intermédiaire qui contredirait la dichotomie.

Ainsi Solecki (dans [So]) a établi que cette dichotomie était vérifiée pour les relations d'égalité modulo un idéal de parties de $\omega$. Il a même établi un résultat plus fort :

Tout idéal $I$, analytique comme partie de $2^{\omega}$, vérifie l'une des deux assertions suivantes:

- $I$ est polonisable (comme groupe $(I, \triangle))$;

- l'idéal $I_{1}$ se plonge en $I$, relation entre idéaux qui est plus forte que le simple plongement borélien ou même continu des relations d'équivalence.

De plus si $I$ est polonisable, il est $\mathrm{F}_{\sigma}$ ou alors vrai $\boldsymbol{\Pi}_{3}^{0}$.

Le présent travail est effectué en essayant de comparer $\mathbb{R}^{\omega}$ et $2^{\omega}$ de la façon suivante : si on identifie les parties de $\omega$ et leurs fonctions caractéristiques qui sont les éléments de $2^{\omega}$, on peut considérer la relation d'infériorité composante par composante dans $\mathbb{R}^{\omega}$ comme une extension de la relation d'inclusion entre parties de $\omega$, la somme dans $\mathbb{R}^{\omega}$ comme une extension de la réunion de parties de $\omega$; l'analogue d'un idéal de $\omega$ sera donc un sous-espace vectoriel de $\mathbb{R}^{\omega}$ clos quand on réduit en valeur absolue les composantes d'un de ses éléments; on dira qu'un tel espace est héréditaire.

On montre ici le même résultat général que Solecki [So] :

(A) Si $X$ est un sous-espace héréditaire de $\mathbb{R}^{\omega}$, analytique comme partie de $\mathbb{R}^{\omega}$, alors on est dans un des deux cas suivants :

- $E_{1} \sqsubseteq_{\mathrm{c}} \mathbb{R}^{\omega} / X$;

- $X$ est polonisable (comme espace vectoriel).

Ici aussi on a quelque chose de plus que $E_{1} \sqsubseteq_{\mathrm{c}} \mathbb{R}^{\omega} / X$ : si $X$ n'est pas polonisable, on a $c_{00} \leq_{\operatorname{lin}} X$ ou $\ell_{\infty} \leq_{\operatorname{lin}} X$, avec les notations usuelles pour l'espace des suites finies et l'espace des suites bornées. (Ici $E \leq_{\text {lin }} F$ signifie qu'il y a une application linéaire continue injective $l: \mathbb{R}^{\omega} \rightarrow \mathbb{R}^{\omega}$ telle que $l^{-1}(F)=E$. Et comme c'est l'usage on note $\mathcal{R} \sqsubseteq_{\mathrm{c}} \mathcal{Q}$ entre relations d'équivalence sur des espaces topologiques $Y, Z$ s'il y a $f: Y \rightarrow Z$ continue injective telle que $\forall y, y^{\prime}, f(y) \mathcal{Q} f\left(y^{\prime}\right) \Leftrightarrow y \mathcal{R} y^{\prime}$.)

Enfin, on a la même précision sur la complexité borélienne possible des sous-espaces polonisables :

(B) Si $X$ est polonisable, alors il est fermé, ou sinon $\boldsymbol{\Sigma}_{2}^{0}$-complet ou $\Pi_{3}^{0}$-complet. 
La première partie est consacrée aux définitions : on vérifie que $E_{1}$ se plonge dans la relation induite par $\ell_{\infty}$ (c'est (1.3.1)). On vérifie aussi la complexité descriptive de $c_{0}, \ell_{\infty}$, qui serviront. Un plongement de $E_{1}$ dans $c_{00}$ est aussi donné au (1.3.1).

La deuxième partie est consacrée à l'étude des "évaluations", analogues pour les suites de réels des sous-mesures pour les parties de $\omega$, et qui permettent de définir de manière canonique des sous-espaces héréditaires polonisables ainsi que leur structure métrique complète séparable.

La dichotomie générale $(\mathrm{A})$ est l'objet du paragraphe 3 : après avoir éliminé dans l'étude un type d'espaces en quelque sorte dégénérés dans le paragraphe 3.1, les espaces n'ayant pas de suites aux composantes toutes non nulles, pour lesquels on trouve toujours un plongement de $c_{00}$, on refait en fait la même étude que Solecki [So] pour les idéaux; on définit dans le paragraphe 3.2 une notion de petitesse pour les parties de $\mathbb{R}^{\omega}$ (et d'une de ses compactifications), et on introduit la dichotomie selon qu'on a ou non une bonne notion de petitesse :

- Si une réunion dénombrable d'ensembles petits peut être grande, ce qui est l'hypothèse (3.3.1), on exhibe dans (3.3.3) un plongement de $c_{00}$ ou de $\ell_{\infty}$ dans $X$.

- Si au contraire une réunion dénombrable d'ensemble petits est un ensemble petit, ce qui est l'hypothèse (3.4.1), on peut utiliser les ensembles grands pour construire par des procédés du type théorie de la mesure une évaluation sur $\mathbb{R}^{\omega}$, et $X$ sera l'espace polonais associé à cette évaluation : c'est la proposition (3.4.15).

Cette dichotomie est résumée dans (3.4.17).

Enfin, on tire de cette dichotomie un certain nombre de résultats sur les espaces qui sont polonisables, essentiellement ce que j'ai résumé dans (B), au paragraphe 4 .

En plus des références relatives à des résultats précis, on se reportera utilement aux ouvrages de A. Kechris [K] et de Moschovakis [M] pour les notions de théories descriptives, ainsi qu'à celui de Schaefer [Sc] pour les propriétés générales des espaces vectoriels ordonnés.

\section{DÉFINITIONS}

1.1. Structure de $\mathbb{R}^{\omega} \cdot \mathbb{R}^{\omega}$ est muni de sa topologie polonaise usuelle, produit de la topologie de $\mathbb{R}$, pour laquelle les $W_{N}$ constituent un système fondamental de voisinages de $0=(0,0,0, \ldots)$, où

$$
W_{N}=\left\{x \in \mathbb{R}^{\omega}|\forall n<N,| x(n) \mid<1 /(N+1)\right\},
$$


et qui correspond à la métrique invariante complète suivante :

$$
d_{\omega}(x, y)=\sum_{n} \frac{\inf (1,|x(n)-y(n)|)}{2^{n}} .
$$

On désignera par l'indice ou l'exposant (pour l'adhérence par exemple) $\omega$ ce qui est relatif à la topologie usuelle de $\mathbb{R}^{\omega}$, notée elle-même $\tau_{\omega}$, ainsi qu'aux topologies induites par $\tau_{\omega}$ sur les parties de $\mathbb{R}^{\omega}$.

On notera $u_{p}$ l'élément de $\mathbb{R}^{\omega}$ dont la $(p+1)$-ème composante (c'est-à-dire $\left.u_{p}(p)\right)$ est égale à 1 et dont les autres composantes sont nulles.

Si $A \subseteq \omega$, on associe à tout $x \in \mathbb{R}^{\omega}$ les suites suivantes :

$$
\pi_{A}(x)=\left((x(k))_{k \in A},(0)_{k \notin A}\right)
$$

est la suite de $\mathbb{R}^{\omega}$ ayant comme composantes celles de $x$ pour les indices qui sont dans $A$ et 0 pour les autres indices, et

$$
\varepsilon_{A}(x)=x-\pi_{A}(x)=\pi_{\omega-A}(x) .
$$

Ces opérations sont les analogues dans $\mathbb{R}^{\omega}$ de celles, pour les suites de 0 et de 1 représentant des parties de $\omega$, consistant à prendre l'intersection avec une partie $A$ fixe pour la première, et à prendre le reste de la suite pour la seconde.

En particulier on a

$$
\begin{aligned}
& \pi_{n}(x)=(x(0), x(1), \ldots, x(n-1), 0,0,0, \ldots), \\
& \varepsilon_{n}(x)=(0,0, \ldots, 0, x(n), x(n+1), \ldots) .
\end{aligned}
$$

Par ailleurs on identifiera couramment une $A$-suite de réels, c'est-à-dire une suite indexée par les éléments de $A$, avec la suite de $\mathbb{R}^{\omega}$ obtenue en complétant par des 0 les composantes manquantes.

1.2. Préordre et espaces héréditaires. On définit sur $\mathbb{R}^{\omega}$ le préordre suivant :

$$
x \leq_{\omega} y \Leftrightarrow \forall n \in \omega,|x(n)| \leq|y(n)| .
$$

On notera aussi $|x|=(|x(n)|)_{n}$ la suite des valeurs absolues des composantes de $x$, et $x \vee y$ et $x \wedge y$ les suites telles que pour tout $k$,

$$
(x \vee y)(k)=\sup (|x(k)|,|y(k)|), \quad(x \wedge y)(k)=\inf (|x(k)|,|y(k)|)) .
$$

Ainsi on a

$$
\left(x \leq_{\omega} y \text { et } y \leq_{\omega} x\right) \Leftrightarrow|x|=|y|, \quad x \leq_{\omega} y \Leftrightarrow|x| \leq_{\omega}|y| .
$$

Notons que ce préordre devient un ordre quand on le restreint à $\left(\mathbb{R}^{+}\right)^{\omega}$ et que l'application $x \mapsto|x|$ est croissante.

On a enfin

$$
\begin{aligned}
& \left(x \leq_{\omega} y \text { et } x^{\prime} \leq_{\omega} y^{\prime}\right) \Rightarrow x+x^{\prime} \leq_{\omega}|y|+\left|y^{\prime}\right|, \\
& \left(x \leq_{\omega} y \text { et }|\lambda| \leq_{|\mu|}\right) \Rightarrow \lambda x \leq_{\omega} \mu y .
\end{aligned}
$$


Définition (1.2.1). Une partie $X$ de $\mathbb{R}^{\omega}$ est dite héréditaire si elle est "close par suites $\leq_{\omega}$-plus petites", c'est-à-dire si

$$
\left(x \in X \text { et } y \leq_{\omega} x\right) \Rightarrow y \in X .
$$

On va s'intéresser dans ce qui suit aux sous-espaces vectoriels de $\mathbb{R}^{\omega}$ qui sont héréditaires, ce qu'on nomme en général des idéaux de $\mathbb{R}^{\omega}$ (cf. $[\mathrm{Sc}]$ ). Pour se borner aux cas significatifs on n'étudiera que les espaces libres :

DÉfinition (1.2.2). Un sous-espace $E$ de $\mathbb{R}^{\omega}$ est libre s'il n'est nul sur aucune composante, c'est-à-dire,

$$
\forall n \in \omega, \exists x \in E, \quad x(n) \neq 0 .
$$

Un espace non libre est soit de dimension finie, auquel cas son étude est triviale pour ce qui nous occupe ici, soit c'est un idéal d'un $\mathbb{R}^{A}$ avec $A$ infini minimal; or $\mathbb{R}^{A}$ est alors isomorphe, composante par composante, à $\mathbb{R}^{\omega}$, et donc l'espace étudié est isomorphe à un espace libre.

Désormais, sauf mention expresse, les espaces considérés seront supposés libres.

Remarque (1.2.3). Si E est un sous-espace héréditaire de $\mathbb{R}^{\omega}$, alors $E$ est libre si et seulement si $c_{00} \subseteq E$.

Preuve. On utilise l'hérédité : si $x \in E$ est tel que $x(n) \neq 0$, comme $x(n) u_{n} \leq_{\omega} x$, on a $x(n) u_{n} \in E$ puis $\mathbb{R} u_{n} \subseteq E$.

On pose aussi, définition qui sera utile dans la suite :

DÉfinition (1.2.4). Si $x \in \mathbb{R}^{\omega}$, on note $C[x]$ l'ensemble des $y$ tels que $y \leq{ }_{\omega} x$.

Propriété (1.2.5). C $[x]$ est un compact héréditaire de $\mathbb{R}^{\omega}$.

Pour comparer les sous-espaces héréditaires, on va utiliser les notions suivantes :

DÉfinition (1.2.6). Si $E$ et $F$ sont deux sous-espaces héréditaires de $\mathbb{R}^{\omega}$, on note $E \leq \leq_{\operatorname{lin}} F$ s'il existe une application linéaire continue injective $f: \mathbb{R}^{\omega} \rightarrow \mathbb{R}^{\omega}$ telle que $f^{-1}(F)=E$. Si on peut choisir $f$ croissante pour $\leq_{\omega}$, on note $E \leq_{\text {lin }}^{+} F$.

On sait que tout espace héréditaire $E$ induit sur $\mathbb{R}^{\omega}$ la relation d'équivalence suivante :

$$
x \equiv_{E} y \Leftrightarrow x-y \in E .
$$

On a donc aussi les relations de hiérarchies classiques entre relations d'équivalence $\equiv_{a}, \equiv_{b}$ sur des espaces topologiques $X, Y$ soit pour celle qui nous intéressent : $\equiv_{a} \sqsubseteq_{\mathrm{c}} \equiv_{b}$ s'il y a $f: X \rightarrow Y$ injective continue telle que

$$
x \equiv_{a} x^{\prime} \Leftrightarrow f(x) \equiv_{b} f\left(x^{\prime}\right) .
$$


On utilisera à l'occasion les notations usuelles où l'indice $\mathrm{B}$ remplace c si on veut juste une $f$ borélienne, et $\leq$ remplace $\sqsubseteq$ si on n'exige plus $f$ injective.

Citons quelques relations particulières : finies.

- $E_{0}$ est la relation sur $2^{\omega}=\operatorname{Pow}(\omega)$ d'égalité modulo les suites (parties)

- $E_{0}^{\omega}$ est la relation sur $2^{\omega^{2}}$ qui dit que $x E_{0}^{\omega} y \Leftrightarrow \forall n, x(n, \cdot) E_{0} y(n, \cdot)$ où $x(n, \cdot)$ désigne la suite des $x(n, k)$ quand $k$ parcourt $\omega$.

- $E_{1}$ est la relation entre parties de $\omega^{2}$ d'égalité modulo l'idéal qui a été défini dans l'introduction : $x E_{1} y \Leftrightarrow \exists n_{0}, \forall n \geq n_{0}, x(n, \cdot)=y(n, \cdot)$.

On notera aussi $(n, k) \mapsto\langle n, k\rangle$ une bijection $\omega^{2} \rightarrow \omega$ fixée une fois pour toutes, au moyen de laquelle on considérera en général que $E_{1}$ et $E_{0}$ sont des relations sur $2^{\omega}$.

Enfin précisons la notion déjà évoquée d'espace polonisable :

DÉfinition (1.2.7). Un groupe topologique $(G, \tau)$ est polonisable s'il existe une topologie polonaise plus fine que $\tau$ et toujours compatible avec la structure de groupe. Un sous-espace $E$ de $\mathbb{R}^{\omega}$ est dit polonisable s'il existe une topologie polonaise $\tau^{\prime}$ sur $E$, plus fine que la restriction de $\tau_{\omega}$ à $E$ et telle que $\left(E, \tau^{\prime}\right)$ soit toujours un espace vectoriel topologique.

1.3. Quelques espaces particuliers. On utilisera quelques sousespaces héréditaires classiques de $\mathbb{R}^{\omega}$ :

$$
\ell_{\infty}=\left\{x \in \mathbb{R}^{\omega}|\exists M>0, \forall n,| x(n) \mid \leq M\right\},
$$

qui est un Banach avec la norme $\|x\|_{\infty}=\sup _{n}|x(n)|$, définition qu'on peut étendre par $\|x\|_{\infty}=+\infty$ si $x \notin \ell_{\infty}$.

$\ell_{\infty}$ est non séparable mais son sous-espace $c_{0}=\left\{x \mid \lim _{n \rightarrow \infty} x(n)=0\right\}$ est Banach séparable avec la norme induite.

Une partie dénombrable dense pour la topologie de $c_{0}$ est $\mathbb{D}=\bigoplus_{n} \mathbb{Q} u_{n}=$ $\bigcup_{n} \mathbb{Q}^{n}$, également dense dans $\mathbb{R}^{\omega}$ pour la topologie produit.

Citons enfin l'espace des suites finies $c_{00}$.

Propriété (1.3.1). $\ell_{\infty}$ est un vrai $\boldsymbol{\Sigma}_{2}^{0}$ et $c_{0}$ est un vrai $\boldsymbol{\Pi}_{3}^{0} ;$ de plus $\ell_{\infty}$ n'est pas polonisable en tant qu'espace vectoriel ou même que groupe additif et

$$
E_{1} \sqsubseteq_{\mathrm{c}} \equiv_{c_{00}}, \quad E_{1} \sqsubseteq_{\mathrm{c}} \equiv_{\ell_{\infty}}, \quad E_{0}^{\omega} \sqsubseteq \equiv_{c_{0}} .
$$

Preuve. Les définitions donnent au plus les classes de Borel indiquées :

$$
\begin{aligned}
x \in \ell_{\infty} & \Leftrightarrow \exists p \in \omega, \forall n \in \omega,|x(n)| \leq p ; \\
x \in c_{0} & \Leftrightarrow \forall p \in \omega, \exists n_{0} \in \omega, \forall n \geq n_{0},|x(n)| \leq 1 /(p+1) .
\end{aligned}
$$

Par ailleurs la relation $\equiv_{E}$ est toujours de la même classe que $E$ (c'est l'image réciproque de $E$ par l'application continue $(x, y) \mapsto x-y$ de $\mathbb{R}^{\omega} \times \mathbb{R}^{\omega}$ 
dans $\mathbb{R}^{\omega}$, donc la classe de $\equiv_{E}$ est inférieure ou égale à la classe de $E$, et par ailleurs $E$ est la classe de 0 , donc de même classe que $\equiv_{E} \cap\{0\} \times \mathbb{R}^{\omega}$ ).

Donc si on montre les plongements indiqués de relations d'équivalence, on obtiendra au moins les classes indiquées puisqu'il s'agit de plongements continus, et que $E_{1}$ et $E_{0}$ sont de vrais $\boldsymbol{\Sigma}_{2}^{0}$, et $E_{0}^{\omega}$ un vrai $\boldsymbol{\Pi}_{3}^{0}$. Tout se ramène donc à ces plongements.

$g: 2^{\omega^{2}} \rightarrow \mathbb{R}^{\omega}$ telle que $g(\alpha)=x \Leftrightarrow[\forall n, k, x(\langle n, k\rangle)=n \alpha(n, k)]$ témoigne que $E_{1} \sqsubseteq_{\mathrm{c}} \equiv_{\ell_{\infty}}$.

Ceci prouve aussi que $\equiv_{\ell_{\infty}}$ n'est plongeable au sens $\leq_{\mathrm{B}}$ dans aucune relation induite par une action de groupe polonaise puisque on sait que $E_{1}$ ne l'est pas; en particulier $\ell_{\infty}$ n'est pas polonisable comme groupe additif.

Enfin $h: 2^{\omega^{2}} \rightarrow \mathbb{R}^{\omega}$ telle que $h(\alpha)=x \Leftrightarrow\left[\forall n, k, x(\langle n, k\rangle)=\frac{1}{n+1} \alpha(n, k)\right]$ témoigne que $E_{0}^{\omega} \sqsubseteq_{\mathrm{c}} \equiv_{c_{0}}$.

Il reste à vérifier que $E_{1} \sqsubseteq_{\mathrm{c}} \equiv_{c_{00}}$; il suffit de prendre par exemple

$$
g: 2^{\omega^{2}} \rightarrow \mathbb{R}^{\omega}, \quad \alpha \mapsto g(\alpha)=\sum_{n}\left(\sum_{\alpha(n, k)=1} 3^{-k}\right) u_{n}
$$

pour obtenir le plongement souhaité.

\section{UNE CONSTRUCTION D'ESPACES HÉRÉDITAIRES POLONISABLES}

2.1. Evaluations. On va maintenant s'intéresser à une classe aisément constructible d'espaces héréditaires polonisables. On construira en même temps leurs topologies propres. Les topologies considérées seront toujours plus fines que $\tau_{\omega}$ puisqu'on veut préserver la structure d'espace de suites, donc d'espace produit. Ainsi une suite convergente dans $E$ convergera vers la même limite dans $\tau_{\omega}$. On va introduire une définition.

DÉfinition (2.1.1). Une évaluation sur $\mathbb{R}^{\omega}$ est une application $\varphi$ : $\mathbb{R}^{\omega} \rightarrow \overline{\mathbb{R}^{+}}=[0,+\infty]$ vérifiant les conditions suivantes :

$\mathrm{Ev}_{1} \quad \varphi$ est positive : $\varphi(x)=0 \Leftrightarrow x=0$;

$\mathrm{Ev}_{2} \varphi$ est sous-additive : $\forall x, y, \varphi(x+y) \leq \varphi(x)+\varphi(y)$;

$\mathrm{Ev}_{3} \varphi$ est croissante : $\forall x, y, x \leq_{\omega} y \Rightarrow \varphi(x) \leq \varphi(y)$;

$\mathrm{Ev}_{4} \quad \varphi$ est "sous-homogène" : $\forall x \in \mathbb{R}^{\omega}, \alpha \geq 1, \varphi(\alpha x) \leq \alpha \varphi(x)$.

$\mathrm{Ev}_{5} \varphi$ est semi-continue inférieurement : si $\left.\left.r \geq 0, \varphi^{-1}(] r,+\infty\right]\right)$ est un $\tau_{\omega}$-ouvert.

$\mathrm{Ev}_{6} \varphi$ est compatible avec les topologies polonaises des sous-espaces finis $\mathbb{R}^{n}$ : sa restriction à chacun de ces espaces est continue.

Remarques. (a) Ev $v_{5}$ est équivalent à l'assertion suivante :

$\mathrm{Ev}_{5}^{\prime} \quad$ pour toute suite $x_{n}$ qui $\tau_{\omega}$-converge vers une limite $x$, on a

$$
\liminf _{n} \varphi\left(x_{n}\right) \geq \varphi(x) .
$$


(b) $\varphi$ est "libre" : $\forall n, \varphi\left(u_{n}\right)<+\infty$, puisque d'après $\mathrm{Ev}_{6}$ on a, pour chaque $n$, un $t$ tel que $\varphi\left(t u_{n}\right)<1$ et donc $\varphi\left(u_{n}\right) \leq(1 / t) \varphi\left(t u_{n}\right)<+\infty$.

On dit que $\varphi$ est finie si $\varphi(x)<+\infty$ pour tout $x$.

On dira que deux évaluations $\varphi$ et $\psi$ sont équivalentes si on a

$$
\begin{aligned}
& \forall \varepsilon>0, \exists \eta>0, \forall x, \quad \varphi(x)<\eta \Rightarrow \psi(x)<\varepsilon, \\
& \forall \varepsilon>0, \exists \eta>0, \forall x, \quad \psi(x)<\eta \Rightarrow \varphi(x)<\varepsilon .
\end{aligned}
$$

Alors toute $\varphi$ est clairement équivalente à une évaluation finie : $\inf (1, \varphi)$.

2.2. Sous-espaces engendrés par une évaluation. Soit $\varphi$ une évalua-

tion et posons $d_{\varphi}(x, y)=\varphi(x-y)$ pour $x, y$ dans $\mathbb{R}^{\omega}$. D'après $\mathrm{Ev}_{1}, \mathrm{Ev}_{2}$ et $\operatorname{Ev}_{3}$ (qui entraîne $\varphi(-x)=\varphi(x)$ ), $d_{\varphi}$ a les propriétés d'une distance, mais qui prendrait la valeur $+\infty$. L'équivalence entre deux évaluations préserve visiblement les topologies engendrées par les boules qu'elles définissent, donc on peut toujours supposer que $\varphi$ est finie. De plus, d'après la définition de $d_{\varphi}$ on a

$$
\forall x, y, z, \quad d_{\varphi}(x+z, y+z)=d_{\varphi}(x, y)
$$

ce qui prouve bien que $d_{\varphi}$ définit une topologie de groupe métrique sur $\left(\mathbb{R}^{\omega},+\right)$. Mais cette topologie n'est pas forcément compatible avec la structure vectorielle :

PropriÉté (2.2.1). d définit une topologie plus fine que $\tau_{\omega}$ sur $\mathbb{R}^{\omega}$, qui est compatible avec la structure vectorielle d'un sous-espace $E$ de $\mathbb{R}^{\omega}$ si et seulement si

$$
\forall x \in E, \quad \lim _{t \rightarrow 0, t \in \mathbb{R}} \varphi(t x)=0,
$$

et ceci est vérifié pour $E=c_{00}$. Enfin $d_{\varphi}$ est complète.

Preuve. 1) Comme $d_{\varphi}$ est compatible avec l'addition, pour s'assurer que $\tau_{\varphi} \supseteq \tau_{\omega}$ il suffit de prouver que les $W_{N}$ sont des $\varphi$-voisinages de 0 .

Soit $r=\inf _{k<N}\left(\varphi\left(u_{k} /(N+1)\right)\right)$. Alors la boule $B_{d_{\varphi}}(0, r)=\{x \mid \varphi(x)$ $<r\}$ est contenue dans $W_{N}$. En effet si $\varphi(x)<r$ on a par croissance $\left(\operatorname{Ev}_{3}\right)$,

$$
\varphi\left(x(k) u_{k}\right) \leq \varphi(x)<r \leq \varphi\left(\frac{u_{k}}{N+1}\right) \quad \text { si } k<N,
$$

donc encore par croissance on a forcément $|x(k)|<1 /(N+1)$ et $x \in W_{N}$.

2) $\mathrm{Si}\left(E, d_{\varphi}\right)$ est un espace vectoriel métrique on a bien évidemment pour $x \in E, \lim _{t \rightarrow 0, t \in \mathbb{R}} t x=0$ dans $\tau_{\varphi}$, c'est-à-dire $\lim _{t \rightarrow 0, t \in \mathbb{R}} \varphi(t x)=0$. Réciproquement, si $\lim _{t \rightarrow 0, t \in \mathbb{R}} \varphi(t x)=0$ pour tout $x \in E$, alors l'application

$$
\mathbb{R} \times \mathbb{R}^{\omega} \rightarrow \mathbb{R}^{\omega}, \quad(\lambda, x) \mapsto \lambda x,
$$


est bien continue car

$$
\begin{aligned}
d_{\varphi}\left(\lambda x, \lambda_{0} x_{0}\right) & =\varphi\left(\lambda x-\lambda_{0} x_{0}\right) \leq \varphi\left(\lambda\left(x-x_{0}\right)\right)+\varphi\left(\left(\lambda-\lambda_{0}\right) x_{0}\right) \\
& \leq \sup (1,|\lambda|) \varphi\left(x-x_{0}\right)+\varphi\left(\left(\lambda-\lambda_{0}\right) x_{0}\right)
\end{aligned}
$$

grâce à la sous-homogénéité et à la croissance (si $|\lambda| \leq 1$ alors $\lambda y \leq_{\omega} y$ ).

Ceci prouve que si $\lambda \rightarrow \lambda_{0}$ (et donc $\lambda$ reste borné) et si $d_{\varphi}\left(x, x_{0}\right) \rightarrow 0$ alors $d_{\varphi}\left(\lambda x, \lambda_{0} x_{0}\right) \rightarrow 0$.

3) $c_{00}$ vérifie la condition précédente à cause de $\mathrm{Ev}_{6}$.

4) Pour montrer la complétude de $d_{\varphi}$ on aura besoin du lemme suivant :

LEMME (2.2.2). Soit $\left(p_{n}\right)$ une suite d'éléments de $\left(\mathbb{R}^{+}\right)^{\omega}$ et $x$ une suite de réels telle que

$$
\forall k, \quad|x(k)| \leq \sum_{n} p_{n}(k)
$$

Alors on a

$$
\varphi(x) \leq \sum_{n} \varphi\left(p_{n}\right)
$$

Preuve. Soit $r<\varphi(x)=\varphi(|x|)$. Par semi-continuité inférieure il y a un $\lambda \in] 0,1\left[\right.$ et un début $\pi_{k_{0}}(|x|)=\left(|x(0)|, \ldots,\left|x\left(k_{0}-1\right)\right|, 0,0, \ldots\right)$ tel que $\varphi\left(\lambda \pi_{k_{0}}(|x|)\right)>r$. Pour chaque composante $k<k_{0}$ on a un $n_{k}$ tel que $\sum_{n<n_{k}} p_{n}(k) \geq \lambda|x(k)|$; si $N$ est supérieur à tous ces $n_{k}$ et $q=\sum_{n<N} p_{n}$ on aura donc $\lambda \pi_{k_{0}}(|x|) \leq_{\omega} q$, d'où

$$
r<\varphi\left(\lambda \pi_{k_{0}}(|x|)\right) \leq \varphi(q) \leq \sum_{n<N} \varphi\left(p_{n}\right) \leq \sum_{n} \varphi\left(p_{n}\right) .
$$

Ceci prouve bien le lemme.

Complétude de $d_{\varphi}$. Maintenant soit $\left(x_{n}\right)_{n}$ une suite $d_{\varphi}$-Cauchy. On suppose, en prenant au besoin une sous-suite, que $d_{\varphi}\left(x_{n}, x_{n+1}\right)<1 / 2^{n}$. Par croissance

$$
\varphi\left(\left(x_{n}(k)-x_{p}(k)\right) u_{k}\right) \leq \varphi\left(x_{n}-x_{p}\right),
$$

donc $\left(x_{n}(k) u_{k}\right)_{n}$ est aussi $d_{\varphi}$-Cauchy.

Or on peut remarquer que

$$
\forall \varepsilon>0, \exists \eta>0, \quad \varphi\left(t u_{k}\right)<\eta \Rightarrow|t|<\varepsilon ;
$$

ceci se vérifie par l'absurde : si c'était faux, il y aurait une suite de réels $t_{j}$ et $r>0$ tels que $\left|t_{j}\right| \geq r$ et $\varphi\left(t_{j} u_{k}\right)$ tend vers 0 . On aurait alors par croissance $\varphi\left(r u_{k}\right) \leq \varphi\left(t_{j} u_{k}\right)$, donc $\varphi\left(r u_{k}\right)=0$, ce qui contredirait la positivité de $\varphi$.

Ceci implique en particulier que la suite de réels $\left(x_{n}(k)\right)_{n}$ est aussi de Cauchy et converge dans $\mathbb{R}$ vers une limite $x(k)$. Posons $p_{n}=\left|x_{n}-x_{n+1}\right|$ dans $\mathbb{R}^{\omega}$. Pour tout $k$ on a clairement

$$
\left|x(k)-x_{n_{0}}(k)\right|=\left|\sum_{n \geq n_{0}}\left(x_{n}(k)-x_{n+1}(k)\right)\right| \leq \sum_{n \geq n_{0}} p_{n}(k) .
$$


Donc d'après le lemme (2.2.2),

$$
\varphi\left(x-x_{n_{0}}\right) \leq \sum_{n \geq n_{0}} \varphi\left(p_{n}\right)<\sum_{n \geq n_{0}} 2^{-n}=2^{1-n_{0}} \rightarrow 0,
$$

ce qui prouve bien que $x$ est la $d_{\varphi}$-limite de $\left(x_{n}\right)_{n}$.

DÉfinition (2.2.3). Fin $(\varphi)$ sera l'ensemble des $x$ tels que $\lim _{\lambda \rightarrow 0} \varphi(\lambda x)$ $=0 . \operatorname{Exh}(\varphi)$ sera l'adhérence de $c_{00}$ dans la topologie $\tau_{\varphi}$.

REMARQUe (2.2.4). (a) Si on n'a pas supposé $\varphi$ finie, elle l'est néanmoins sur $\operatorname{Fin}(\varphi)$ : en effet, si $x \in \operatorname{Fin}(\varphi)$ il y a $t$ tel que $\varphi(t x)<1$, donc $\varphi(x) \leq$ $\sup (1,1 / t) \varphi(t x)<+\infty$.

(b) Ces espaces ne changent pas si on remplace $\varphi$ par une évaluation équivalente au sens précisé plus haut (cette notion signifie précisément qu'il est identique de converger vers 0 pour l'une ou l'autre, et que les distances engendrées sont équivalentes) ; l'hypothèse qu'on travaille avec une évaluation finie n'est donc nullement restrictive.

Propriété (2.2.5). • Fin $(\varphi)$ est un sous-espace héréditaire et $d_{\varphi}$ est complète sur $\operatorname{Fin}(\varphi)$, et compatible avec sa structure vectorielle.

- $\operatorname{Exh}(\varphi)$ est un sous-espace héréditaire de $\operatorname{Fin}(\varphi)$ et $d_{\varphi}$ est séparable complète sur $\operatorname{Exh}(\varphi) ; \mathbb{D}$ est dénombrable dense dans $\operatorname{Exh}(\varphi)$ et

$$
x \in \operatorname{Exh}(\varphi) \Leftrightarrow \lim _{n} \varphi\left(\varepsilon_{n}(x)\right)=0 .
$$

Preuve. (1) Si $\alpha, \beta$ sont réels et $x, y \in \mathbb{R}^{\omega}$ alors pour tout réel $t$ on a

$$
\varphi(t(\alpha x+\beta y)) \leq \sup (1,|\alpha|) \varphi(t x)+\sup (1,|\beta|) \varphi(t y)
$$

et si $x \leq_{\omega} y$ alors $\varphi(t x) \leq \varphi(t y)$. On voit donc aisément que $\operatorname{Fin}(\varphi)$ est un sous-espace héréditaire.

(2) Pour montrer que $d_{\varphi}$ reste complète restreinte à $\operatorname{Fin}(\varphi)$, il suffit de montrer que cet espace est $d_{\varphi}$-fermé. Soit donc $\left(x_{n}\right)_{n}$ dans $\operatorname{Fin}(\varphi)$ convergeant pour $d_{\varphi}$ vers $x$. Montrons que $x \in \operatorname{Fin}(\varphi)$ :

Soit $\varepsilon>0$; on a un $n$ tel que $\varphi\left(x-x_{n}\right)<\varepsilon / 2$. Comme $x_{n} \in \operatorname{Fin}(\varphi)$, on peut trouver un $\eta<1$ tel que $|t|<\eta \Rightarrow \varphi\left(t x_{n}\right)<\varepsilon / 2$. Alors si $|t|<\eta$ on a $\varphi(t x) \leq \varphi\left(t\left(x-x_{n}\right)\right)+\varphi\left(t x_{n}\right) \leq \sup (1,|t|) \varphi\left(x-x_{n}\right)+\varphi\left(t x_{n}\right)<\frac{\varepsilon}{2}+\frac{\varepsilon}{2}=\varepsilon$.

(3) $c_{00} \subseteq \operatorname{Fin}(\varphi)$ donc $\bar{c}_{00}^{\varphi}=\operatorname{Exh}(\varphi)$ est un sous-espace $d_{\varphi}$-fermé de l'espace fermé $\operatorname{Fin}(\varphi)$. Ceci prouve que $\left(\operatorname{Exh}(\varphi), d_{\varphi}\right)$ est un sous-espace complet. C'est un sous-espace vectoriel en tant qu'adhérence d'un sousespace vectoriel.

Si $x \in \operatorname{Exh}(\varphi)$ et $\varepsilon>0$, on a $y \in c_{00}$ tel que $\varphi(x-y)<\varepsilon$. Il y a $n$ tel que $y \in \mathbb{R}^{n}$, donc $\varepsilon_{n}(y)=0$ et comme $\varepsilon_{n}(x-y) \leq_{\omega} x-y$, on a

$$
\varphi\left(\varepsilon_{n}(x)\right)=\varphi\left(\varepsilon_{n}(x-y)\right) \leq \varphi(x-y)<\varepsilon .
$$


Donc 0 est valeur d'adhérence de la suite $\left(\varphi\left(\varepsilon_{n}(x)\right)\right)_{n}$ et comme cette suite est décroissante (car la suite $\left(\varepsilon_{n}(x)\right)_{n}$ est $\leq_{\omega}$-décroissante) on a bien $\varphi\left(\varepsilon_{n}(x)\right) \rightarrow 0$.

Réciproquement, si $\varphi\left(\varepsilon_{n}(x)\right) \rightarrow 0$, alors $\pi_{n}(x) \rightarrow x$ pour $d_{\varphi} ;$ or $\pi_{n}(x) \in$ $c_{00}$, donc on a bien $x \in \overline{c_{00}} \varphi$.

Ceci prouve que $\operatorname{Exh}(\varphi)$ est héréditaire puisque si $y \in \operatorname{Exh}(\varphi)$, on a

$$
x \leq_{\omega} y \Rightarrow \varphi\left(\varepsilon_{n}(x)\right) \leq \varphi\left(\varepsilon_{n}(y)\right) \rightarrow 0 .
$$

Enfin $\mathbb{Q}^{n}$ est $\tau_{\omega}$-dense dans $\mathbb{R}^{n}$. Comme $\varphi$ est continue sur cet espace, pour toute suite $x \in \mathbb{R}^{n}$ et tout $\varepsilon>0$, on a un $y \in \mathbb{Q}^{n}$ tel que $\varphi(x-y)<\varepsilon$, ce qui prouve que $\mathbb{Q}^{n}$ est $d_{\varphi}$-dense dans $\mathbb{R}^{n}$ et donc $\mathbb{D}$ dans $c_{00}$. Donc $\mathbb{D}$ l'est aussi dans $\operatorname{Exh}(\varphi)=\overline{c_{00}} \varphi$.

La démonstration est donc complète.

On a ainsi un moyen de construire toute une classe d'espaces polonisables héréditaires. On verra qu'en fait c'est la forme générale de ces espaces.

\section{LA DICHOTOMIE GÉNÉRALE}

Dans cette section, $X$ désignera un espace héréditaire de $\mathbb{R}^{\omega}$ qu'on suppose libre, et analytique comme partie de $\mathbb{R}^{\omega}$.

\subsection{Espaces impropres}

DÉfinition (3.1.1). Un espace héréditaire $X$ est dit propre s'il existe un élément $x \in X$ dont les composantes sont toutes non nulles. Sinon il est dit impropre.

Un exemple type d'espaces impropres est $c_{00}$; il est libre (c'est le plus petit, (1.2.3)) mais n'a aucun élément aux composantes toutes non nulles.

De tels espaces sont non polonisables : si $X$ est polonisable et libre, on a pour tout $k$ un $h(k)>0$ tel que $d_{X}\left(0, h(k) u_{k}\right)<2^{-k}$; alors forcément $h=\sum_{k} h(k) u_{k} \in X$ car $d_{X}$ est complète, et en même temps les composantes de $h$ sont non nulles. En fait, de tels $X$ ne sont même pas complètement métrisables.

On va voir que pour ces espaces, il y a un plongement aisé de $E_{1}$; on se base sur le théorème de Talagrand $[\mathrm{T}]$ pour les idéaux : Si $\mathcal{J}$ est un idéal sur $\omega$ qui est Baire-mesurable comme partie de $2^{\omega}$ (en particulier s'il est analytique ou coanalytique), et s'il est libre $(\forall n,\{n\} \in \mathcal{J})$ et non trivial $(\omega \notin \mathcal{J})$, alors on peut trouver une partition $\left\{F_{n}\right\}_{n}$ de $\omega$ en parties finies telle que

$$
\forall A \subseteq \omega, \quad \bigcup_{n \in A} F_{n} \in \mathcal{J} \Leftrightarrow A \text { est fini. }
$$


Proposition (3.1.2). Si $X$ est impropre, alors $c_{00} \leq_{\operatorname{lin}}^{+} X$ et donc $E_{1} \sqsubseteq_{\mathrm{c}} \equiv_{X}$.

Preuve. Considérons l'ensemble $\mathcal{I} \subseteq 2^{\omega}$ suivant :

$$
A \in \mathcal{I} \Leftrightarrow \exists x \in X, \forall k \in A, x(k) \neq 0 .
$$

C'est un idéal d'après les hypothèses sur $X$. Il ne contient pas $\omega$ puisque $X$ est impropre. Enfin $\mathcal{I}$ est analytique car $X$ l'est. Le théorème de Talagrand [T] fournit une partition de $\omega$ en parties finies $\left(F_{n}\right)_{n}$ comme décrites plus haut. Mais alors la fonction

$$
f: \mathbb{R}^{\omega} \rightarrow \mathbb{R}^{\omega}, \quad x \mapsto f(x)=\sum_{n}\left(x(n) \sum_{k \in F_{n}} u_{k}\right),
$$

est linéaire continue et $\leq_{\omega}$-croissante, envoie $c_{00}$ dans $c_{00} \subseteq X$, et un $x \notin c_{00}$ sur un $f(x)$ tel que $\{k \mid f(x)(k) \neq 0\}=\bigcup_{x(n) \neq 0} F_{n} \notin \mathcal{I}$, donc $f(x) \notin X$. On a donc bien $c_{00} \leq_{\operatorname{lin}}^{+} X$.

On a établi en (1.3.1) que $E_{1} \sqsubseteq_{\mathrm{c}} \equiv_{c_{00}}$, la preuve est donc achevée.

La dichotomie est donc vérifiée pour les espaces impropres : ils sont non polonisables et $E_{1}$ se plonge dedans.

Dans le reste de la section on supposera donc qu'on considère des espaces propres.

3.2. Ensembles petits et grands. On poursuit ici l'analogie entre idéaux vectoriels de $\mathbb{R}^{\omega}$ et idéaux ensemblistes de $2^{\omega}$. On introduit donc une notion de petitesse semblable à celle de Solecki [So] pour les parties de $2^{\omega}$.

DÉfinition (3.2.1). Un ensemble $A \subseteq \mathbb{R}^{\omega}$ est dit petit si on peut trouver une famille dénombrable de fermés $F_{n} \subseteq \mathbb{R}^{\omega}$ et $x \in X$ tels que :

- $F_{n}$ est héréditaire et $\bigcup_{n} F_{n} \supseteq A$;

- pour tout $n, F_{n} \cap C[x]$ est maigre pour la topologie compacte induite $\operatorname{par} \tau_{\omega}$ sur $C[x]$.

Si $A$ est héréditaire et non petit, on dit qu'il est grand.

Quand c'est précisé, le caractère héréditaire des ensembles est nécessaire, car des ensembles maigres, même très réduits, peuvent être non petits : ainsi tout fermé héréditaire $F_{n}$ contenant le singleton $\{x\}$ contient $C[x]$; si par exemple $x=(1,2,3,4, \ldots)$, alors $\{x\}$ n'est pas petit par rapport à $\ell_{\infty}$.

Ainsi un tel $\{x\}$ n'est ni "grand" (il n'est pas héréditaire) ni petit.

En revanche un ensemble héréditaire $A$ est soit grand soit petit par définition.

On va maintenant prouver un certain nombre de propriétés de cette notion. 
PropriÉTÉ (3.2.2). (i) Si B est héréditaire et $B \cap C[x]$ est maigre pour la topologie compacte induite par $\tau_{\omega}$ sur $C[x]$, alors $B \cap C[y]$ est aussi maigre dans $C[y]$ pour tout $y$ tel que $x \leq_{\omega} y$.

(ii) Les ensembles petits forment un idéal de parties de $\mathbb{R}^{\omega}$.

Preuve. (i) Supposons qu'il y ait $y$ tel que $x \leq_{\omega} y$ et $B \cap C[y]$ soit non maigre dans $C[y]$.

Considérons l'ensemble $Z \subseteq \omega$ des $k$ tels que $x(k) \neq 0$; alors $B \cap C\left[\pi_{Z}(y)\right]$ est non maigre dans $C\left[\pi_{Z}(y)\right]$. En effet, on peut voir $C[y]$ comme l'espaceproduit $C\left[\pi_{Z}(y)\right] \times C\left[\varepsilon_{Z}(y)\right]$, et $B \cap C\left[\pi_{Z}(y)\right]$ est alors la projection de $B$ sur $C\left[\pi_{Z}(y)\right]$ car par hérédité tout point de cette projection est dans $B$. Si $B \cap C[y]$ n'est pas maigre, le théorème de Kuratowski-Ulam (cf. par exemple $[\mathrm{K}], \S 8-\mathrm{K}$, p. 53) nous donne que $B \cap C\left[\pi_{Z}(y)\right]$ est aussi non maigre dans $C\left[\pi_{Z}(y)\right]$.

On a pour $n \in Z$ un $\lambda(n) \in] 0,1[$ tel que $x(n)=\lambda(n) y(n)$. Alors l'application

$$
f: C\left[\pi_{Z}(y)\right] \rightarrow C[x]=C\left[\pi_{Z}(x)\right], \quad t \mapsto f(t)=\left((\lambda(n) t(n))_{n \in Z},(0)_{n \notin Z}\right),
$$

est un homéomorphisme de $C\left[\pi_{Z}(y)\right]$ sur $C[x]$ tel que $f(B) \subseteq B$ par hérédité, puisque $f(t) \leq_{\omega} t$. Donc $B \cap C[x]$ n'est pas maigre dans $C[x]$.

(ii) Une sous-partie d'une partie petite reste clairement petite.

Soient $A, A^{\prime}$ deux parties petites et $\left\{F_{n}\right\}_{n},\left\{F_{n}^{\prime}\right\}_{n}$ des fermés héréditaires, et $x, x^{\prime} \in X$ associés par la définition. Alors si $z=x \vee x^{\prime}$, on vérifie aisément, en appliquant (i) à $C[z]$ d'un côté, et à $C[x]$ ou $C\left[x^{\prime}\right]$ de l'autre, que la famille $\operatorname{des} F_{n}$ et des $F_{n}^{\prime}$, et $z$, témoignent que $A \cup A^{\prime}$ est petite.

On va maintenant préciser des propriétés propres aux fermés héréditaires.

Propriété (3.2.3). Soit $F$ un fermé héréditaire de $\mathbb{R}^{\omega}$.

(i) Les assertions suivantes sont équivalentes:

(a) F est petit;

(b) il existe $x \in X$ tel que $F \cap C[x]$ est rare dans $C[x]$.

(ii) Les assertions suivantes sont équivalentes:

(a) F est grand;

(b) $\forall x \in X, \exists n>0, n^{-1} \pi_{n}(x)+\varepsilon_{n}(x) \in F$.

(iii) Les assertions suivantes sont équivalentes :

(a) $F \cap C[x]$ est maigre dans $C[x]$;

(b) pour tout $n \in \omega$, il y a $m>n$ tel que $n^{-1} \pi_{n}(x)+\pi_{[n, m[}(x) \notin F$;

(c) pour toute suite $\Lambda \in([0,1])^{\omega}$, et tout $n \in \omega$, il y a $m>n$ tel que $\pi_{n}(\Lambda) * x+\pi_{[n, m[}(x) \notin F$

(d) pour toute suite $\Lambda \in([0,1])^{\omega}$, il y a une suite $\left(n_{k}\right)_{k}$ strictement croissante telle que $\varepsilon_{\left[n_{k}, n_{k+1}[\right.}(\Lambda) * x+\pi_{\left[n_{k}, n_{k+1}[\right.}(x) \notin F$. 
Preuve. (i) (a) $\Rightarrow$ (b) : Si $F$ est petit, il y a des fermés héréditaires $G_{n}$ et $x \in X$ garantis par (3.2.1) ; alors pour tout $n, G_{n} \cap C[x]$ est rare dans $C[x]$, et $\bigcup_{n} G_{n} \supseteq F$, donc par le théorème de Baire (dans le compact $C[x]$ par exemple), $F \cap C[x]$ est rare dans $C[x]$.

(b) $\Rightarrow$ (a) : Si $F$ et $x \in X$ sont comme dans l'énoncé, la famille $\left\{F_{n}\right\}_{n}$ telle que pour tout $n, F_{n}=F$ et $x$ témoignent bien que $F$ est petit.

(ii) $(\mathrm{a}) \Rightarrow(\mathrm{b}): \mathrm{Si} F$ est grand, on utilise le (i) : $F \cap C[x]$ est non rare et contient donc un ouvert relatif, donc aussi un ouvert de base non vide de la forme $\{t|\forall k<p| t,(k)-a(k) \mid<r\} \cap C[x]$ pour des $p,(a(0), \ldots, a(p-1))$ et $r>0$ donnés.

Il y a donc un $n>p$ tel que $n^{-1}|x(k)|<a(k)+r$ pour tout $k<p$.

Par hérédité, $n^{-1} \pi_{n}(x)+\varepsilon_{n}(x)$ étant $\leq_{\omega}$-majoré par un élément de l'ouvert $\{t|\forall k<p| t,(k)-a(k) \mid<r\} \cap C[x]$, est dans $F$.

$(\mathrm{b}) \Rightarrow(\mathrm{a})$ : Si la deuxième condition est vérifiée, pour tout $x \in X, F \cap C[x]$ contient un ensemble $C\left[n^{-1} \pi_{n}(x)+\varepsilon_{n}(x)\right]$ qui est non rare dans $C[x]$.

(iii) Les trois dernières conditions sont facilement équivalentes, on le voit en utilisant l'hérédité de $F$ et les relations

$$
n^{-1} \pi_{n}(x)+\pi_{[n, p[}(x) \leq_{\omega} n^{-1} \pi_{m}(x)+\pi_{[m, p[}(x) \leq_{\omega} \Lambda * \pi_{m}(x)+\pi_{[m, p[}(x)
$$

pour $m$ et $\Lambda$ fixés, si $n>m$ est assez grand et $p>n$ quelconque.

On montre $(\mathrm{a}) \Leftrightarrow(\mathrm{b})$ :

$(\mathrm{a}) \Rightarrow(\mathrm{b}): m$ existe ; sinon, $F$ étant fermé, on aurait

$$
\lim _{m}\left(n^{-1} \pi_{n}(x)+\pi_{[n, m[}(x)\right)=n^{-1} \pi_{n}(x)+\varepsilon_{n}(x) \in F ;
$$

donc par hérédité $C\left[n^{-1} \pi_{n}(x)+\varepsilon_{n}(x)\right] \subseteq F$ or cet ensemble est d'intèrieur non vide dans $C[x] \subseteq F$.

Non $(\mathrm{a}) \Rightarrow$ Non (b) : Si $F$ n'est pas maigre il contient un ouvert non vide, donc un ensemble du type $\left(a+W_{N}\right) \cap C[x]$ avec $a \in \mathbb{R}^{N} \cap C[x]$. Par hérédité $F$ contient alors $W_{N} \cap C[x]$, ce qui veut dire qu'il y a un $n>N$ assez grand pour que $n^{-1} \pi_{N}(x)+\varepsilon_{N}(x) \in F$. On aura alors si $m>n$,

$$
n^{-1} \pi_{n}(x)+\varepsilon_{[n, m[}(x) \leq_{\omega} n^{-1} \pi_{N}(x)+\varepsilon_{[N, m[}(x) \in F,
$$

donc on a bien Non (b).

On va voir maintenant, en vue du résultat annoncé, que la bonne dichotomie est : les ensembles héréditaires grands sont-ils vraiment "consistants" ou non : peut-on recouvrir un ensemble grand par une réunion dénombrable d'ensembles petits ?

Dans le cas où c'est oui, on verra que $E_{1}$ se plonge continûment dans $\equiv_{X}$, et même qu'on a une "sous-dichotomie" : c'est $c_{00}$ ou $\ell_{\infty}$ qui se plonge dans $X$. 
Dans le cas contraire, on remontera à une famille dénombrable "fondamentale" $\left\{F_{k}\right\}_{k}$, puis à une $\varphi$ pour laquelle les $F_{k}$ extrapolent les voisinages de 0 .

3.3. Le cas non polonisable : plongements de non polonisables canoniques. On se place ici dans le cas où il y a une suite de fermés héréditaires petits dont la réunion est grande, en remarquant qu'une réunion d'ensembles héréditaires est héréditaire.

Hypothèse (3.3.1). Il y a une famille $\left\{A_{n}\right\}_{n \in \omega}$ de parties de $\mathbb{R}^{\omega}$ et des $x_{n} \in X$ tels que :

- $A_{n}$ est fermé héréditaire ;

- $A_{n} \cap C\left[x_{n}\right]$ est maigre dans $C\left[x_{n}\right]$;

- $\bigcup_{n} A_{n}$ est un ensemble grand.

Notations (3.3.2). $u$ désignera un élément de $X$ dont toutes les composantes sont strictement positives, ce qui est possible car $X$ est propre.

Proposition (3.3.3). Sous l'hypothèse (3.3.1) une des deux assertions suivantes est vérifiée:

- $c_{00} \leq_{\operatorname{lin}}^{+} X$;

- $\ell_{\infty} \leq_{\operatorname{lin}}^{+} X$.

De plus dans les deux cas on a $E_{1} \sqsubseteq_{\mathrm{c}} \equiv_{X}$.

Preuve. Soient donc les $\left\{A_{n}\right\}_{n}$ et les $\left(x_{n}\right)_{n}$ donnés par (3.3.1). Sans changer les propriétés de (3.3.1) on peut remplacer chaque $x_{n}$ par $u \vee x_{n}$ et donc on peut supposer (ce qu'on fera dans la suite) que

$$
\forall n, \quad u \leq_{\omega} x_{n} \in\left(\mathbb{R}_{+}^{*}\right)^{\omega} .
$$

Notons tout d'abord que d'après (3.2.3) si on choisit un des $x_{n}$, un $P \in \omega$ et un rang $N \in \omega$, on aura un $M>N$ tel que $P^{-1} \pi_{N}(u)+\pi_{[N, M[}\left(x_{n}\right) \notin A_{n}$.

On peut effectivement appliquer (3.2.3). Comme pour tout $k$ on a

$$
0<u(k) \leq x_{n}(k),
$$

on a bien $\Lambda(k) \in] 0,1]$ tel que $P^{-1} u(k)=\Lambda(k) x_{n}(k)$.

On peut donc construire par récurrence sur le nombre $\langle n, p\rangle$ une suite $N_{p}^{n}$ d'entiers telle que :

- $N_{0}^{0}=0$;

- $\langle n, p\rangle<\langle m, q\rangle \Rightarrow N_{p}^{n}<N_{q}^{m}$;

- $\forall n, p,\langle n, p\rangle+1=\left\langle n^{\prime}, p^{\prime}\right\rangle \Rightarrow\left(N_{p}^{n}+1\right)^{-1} \pi_{N_{p}^{n}}(u)+\pi_{\left[N_{p}^{n}, N_{p^{\prime}}^{n^{\prime}}[\right.}\left(x_{n}\right) \notin A_{n}$. 
Lemme (3.3.4). Soit $B \subseteq \omega^{2}$. Si $\{p \mid(n, p) \in B\}=B_{n}$ est infini pour tout $n$, alors

$$
\sum_{(n, p) \in B} \pi_{\left[N_{p}^{n}, N_{p^{\prime}}^{n^{\prime}}\left(x_{n}\right) \notin X\right.}
$$

(où on convient désormais que pour chaque $(n, p),\left(n^{\prime}, p^{\prime}\right)$ est l'unique couple tel que $\left.\langle n, p\rangle+1=\left\langle n^{\prime}, p^{\prime}\right\rangle\right)$.

Si $B \in I_{1}$, c'est-à-dire $B_{n}=\emptyset$ pour $n$ suffisamment grand, alors

$$
\sum_{(n, p) \in B} \pi_{\left[N_{p}^{n}, N_{p^{\prime}}^{n^{\prime}}[\right.}\left(x_{n}\right) \in X
$$

Preuve. La deuxième assertion est vraie car

$$
B \in I_{1} \Rightarrow \sum_{(n, p) \in B} \pi_{\left[N_{p}^{n}, N_{p^{\prime}}^{n^{\prime}}[\right.}\left(x_{n}\right) \leq_{\omega} \sum_{n<n_{0}, p} \pi_{\left[N_{p}^{n}, N_{p^{\prime}}^{n^{\prime}}\right]}\left(x_{n}\right) \leq_{\omega} \sum_{n<n_{0}} x_{n} \in X .
$$

Pour la première assertion, raisonnons par l'absurde : soit $B$ tel que pour tout $n, B_{n}$ est infini et qu'on ait en même temps

$$
S=\sum_{(n, p) \in B} \pi_{\left[N_{p}^{n}, N_{p^{\prime}}^{n^{\prime}}[\right.}\left(x_{n}\right) \in X,
$$

et posons $T=S+u \in X$.

Soient $n$ fixé et $N \in \omega$. Comme $B_{n}$ est infini, il y a $p \in B_{n}$ tel que $N_{p}^{n}>N$. Alors

$$
\frac{1}{N_{p}^{n}+1} \pi_{N_{p}^{n}}(u)+\pi_{\left[N_{p}^{n}, N_{p^{\prime}}^{n^{\prime}}\right.}\left(x_{n}\right) \notin A_{n} .
$$

Or on a

$$
\frac{1}{N_{p}^{n}+1} \pi_{N_{p}^{n}}(u)+\pi_{\left[N_{p}^{n}, N_{p^{\prime}}^{n^{\prime}[}\right.}\left(x_{n}\right) \leq_{\omega} \frac{1}{N_{p}^{n}+1} \pi_{N_{p}^{n}}(T)+\pi_{\left[N_{p}^{n}, N_{p^{\prime}}^{n^{\prime}}\right.}(T),
$$

donc aussi par hérédité de $A_{n}$,

$$
\frac{1}{N_{p}^{n}+1} \pi_{N_{p}^{n}}(T)+\pi_{\left[N_{p}^{n}, N_{p^{\prime}}^{n^{\prime}}[\right.}(T) \notin A_{n} .
$$

Si on remarque que pour $M=N_{p^{\prime}}^{n^{\prime}}>N_{p}^{n}>N$,

$$
\frac{1}{N_{p}^{n}+1} \pi_{N_{p}^{n}}(T)+\pi_{\left[N_{p}^{n}, N_{p^{\prime}}^{n^{\prime}}[\right.}(T) \leq_{\omega} \frac{1}{N} \pi_{N}(T)+\pi_{[N, M[}(T),
$$

on a aussi

$$
\frac{1}{N} \pi_{N}(T)+\pi_{[N, M[}(T) \notin A_{n} .
$$

Ainsi pour tout $N$, il existe $M>N$ tel que $N^{-1} \pi_{N}(T)+\pi_{[N, M[}(T) \notin X$, ce qui d'après (3.2.3) prouve que $A_{n} \cap C[T]$ est maigre dans $C[T]$. 
Chaque $A_{n} \cap C[T]$ est donc rare dans $C[T]$, ce qui témoigne par définition que l'ensemble $\bigcup_{n} A_{n}$ est petit. Or $\bigcup_{n} A_{n}$ est grand suivant (3.3.1) : une contradiction.

Preuve de (3.3.3) ( fin). Posons $\chi_{n}=\sum_{p} \pi_{\left[N_{p}^{n}, N_{p^{\prime}}^{n^{\prime}}[\right.}\left(x_{n}\right)$; on a $\chi_{n} \leq_{\omega} x_{n}$, donc $\chi_{n} \in X$, et d'après (3.3.4), $\sum_{n} \chi_{n} \notin X$ (cela correspond à $B=\omega^{2}$ ).

Considérons les parties $G \subseteq \omega$ telles qu'il existe $t \in \mathbb{R}^{\omega}$ vérifiant :

- $\forall n \in G, t(n)>0$

- $\sum_{n} t(n) \chi_{n} \in X$.

Ces parties forment visiblement un idéal $\mathcal{I}$ (grâce à l'hérédité de $X$ notamment), et cet idéal est analytique :

$$
G \in \mathcal{I} \Leftrightarrow \exists t \in \mathbb{R}^{\omega},(\forall n, n \notin G \text { ou } t(n)>0) \text { et } \sum_{n} t(n) \chi_{n} \in X ;
$$

or la fonction $t \mapsto \sum_{n} t(n) \chi_{n}$ est continue, donc la dernière condition est analytique en $t$ et $\mathcal{I}$ est $\boldsymbol{\Sigma}_{1}^{1}$.

On a maintenant deux cas distincts :

$1^{\text {er }}$ CAS : $\omega \notin \mathcal{I}$. Alors d'après le théorème de Talagrand $[\mathrm{T}]$, on peut trouver une partition de $\omega$ en sous-ensembles finis $F_{n}$ tels que $H \subseteq \omega$ est fini $\Leftrightarrow \bigcup_{n \in H} F_{n} \in \mathcal{I}$. L'application

$$
f: \mathbb{R}^{\omega} \rightarrow \mathbb{R}^{\omega}, \quad t \mapsto f(t)=\sum_{n} t(n)\left(\sum_{k \in F_{n}} \chi_{k}\right),
$$

est une injection linéaire continue $\leq_{\omega}$-croissante et vérifie :

- Si $t \notin c_{00},|f(t)|=\sum_{n}|t(n)|\left(\sum_{k \in F_{n}} \chi_{k}\right)$ est une combinaison linéaire des $\chi_{k}$ à coefficients positifs, et non nuls si $k \in F_{n}$ pour une infinité de $n$. Par définition de $\mathcal{I}, f(t) \notin X$.

- Si $t \in c_{00}, f(t) \in X$ car c'est une somme finie de termes du type $\sum_{k \in F_{n}} \chi_{k}$, eux-mêmes combinaisons linéaires finies des $\chi_{k}$.

Finalement, $f$ prouve bien que $c_{00} \leq_{\operatorname{lin}}^{+} X$.

$2^{\text {ème }}$ CAS. $\omega \in \mathcal{I}$, c'est-à-dire qu'il y a une suite $e \in\left(\mathbb{R}_{+}^{*}\right)^{\omega}$ telle que $\sum_{n} e(n) \chi_{n} \in X$. Considérons l'application $f$ suivante :

$$
f: \mathbb{R}^{\omega} \rightarrow \mathbb{R}^{\omega}, \quad t \mapsto f(t)=\sum_{n}\left(e(n) \sum_{p} t(p) \pi_{\left[N_{p}^{n}, N_{p^{\prime}}^{n^{\prime}}\right.}\left(x_{n}\right)\right) .
$$

$f$ est de nouveau linéaire continue $\leq_{\omega}$-croissante. Si $t \in \ell_{\infty}$, on a $f(t) \in X$ car

$$
f(t)=\sum_{n}\left(e(n) \sum_{p} t(p) \pi_{\left[N_{p}^{n}, N_{p^{\prime}}^{n^{\prime}}[\right.}\left(x_{n}\right)\right)
$$




$$
\begin{aligned}
& \leq_{\omega}\|t\|_{\infty} \sum_{n}\left(e(n) \sum_{p} \pi_{\left[N_{p}^{n}, N_{p^{\prime}}^{n^{\prime}}[\right.}\left(x_{n}\right)\right) \\
& \leq_{\omega}\|t\|_{\infty} \sum_{n} e(n) \chi_{n} \in X .
\end{aligned}
$$

Si $t \notin \ell_{\infty}$, soient $B=\{(n, p)|| e(n) t(p) \mid \geq 1\} \subseteq \omega^{2}$. Comme $t$ n'est pas bornée, $B_{n}$ est infini pour tout $n$. Donc par le lemme (3.3.4),

$$
\sum_{(n, p) \in B} \pi_{\left[N_{p}^{n}, N_{p^{\prime}}^{n^{\prime}}[\right.}\left(x_{n}\right) \notin X .
$$

Finalement, on a

$$
\begin{aligned}
\sum_{(n, p) \in B} \pi_{\left[N_{p}^{n}, N_{p^{\prime}}^{n^{\prime}}[\right.}\left(x_{n}\right) & \leq \omega \\
& \sum_{(n, p) \in B}\left(e(n) t(p) \pi_{\left[N_{p}^{n}, N_{p^{\prime}}^{n^{\prime}}\right.}\left(x_{n}\right)\right) \\
& \sum_{(n, p) \in \omega^{2}}\left(e(n) t(p) \pi_{\left[N_{p}^{n}, N_{p^{\prime}}^{n^{\prime}}[\right.}\left(x_{n}\right)\right)=f(t) .
\end{aligned}
$$

Ceci prouve que $f(t) \notin X$. Finalement, $f$ témoigne bien que $\ell_{\infty} \leq_{\operatorname{lin}}^{+} X$.

On a déjà vu que $E_{1}$ se plonge continûment dans $\equiv_{c_{00}}$ et dans $\equiv_{\ell_{\infty}}$, donc la preuve est achevée.

3.4. Le cas polonisable : construction d'une évaluation. On va supposer maintenant que (3.3.1) est en défaut : cela signifie que la réunion de toute famille dénombrable de fermés héréditaires petits est un ensemble petit. On est donc sous :

Hypothèse (3.4.1). Si la famille $\left\{A_{n}\right\}_{n \in \omega}$ de parties de $\mathbb{R}^{\omega}$ et les $x_{n} \in X$ vérifient :

- $A_{n}$ est fermé héréditaire;

- $A_{n} \cap C\left[x_{n}\right]$ est maigre dans $C\left[x_{n}\right]$,

alors $\bigcup_{n} A_{n}$ est un ensemble petit.

PropriÉté (3.4.2). L'hypothèse (3.4.1) est équivalente à l'hypothèse (3.4.1') ci-dessous.

Нypothèse $\left(3.4 .1^{\prime}\right)$. Une réunion dénombrable d'ensembles petits est un ensemble petit.

Preuve. $\left(3.4 .1^{\prime}\right) \Rightarrow(3.4 .1)$ est clair.

(3.4.1) $\Rightarrow\left(3.4 .1^{\prime}\right)$ : Soit $\left\{P_{n}\right\}_{n}$ une famille d'ensembles petits. Pour chaque $n$ il y a une famille $\left\{A_{n}^{k}\right\}_{k}$ et $x_{n} \in X$ témoignant que $P_{n}$ est petit. On peut appliquer (3.4.1) aux $A_{n}^{k}$, chacun muni de $x_{n}$, et on obtient que $\bigcup_{n} P_{n}$ est inclus dans un ensemble petit, donc petit. 
On va maintenant se placer dans une compactification de $\mathbb{R}^{\omega}$, à savoir $[-\infty,+\infty]^{\omega}$.

DÉfinition (3.4.3). On notera $\mathbb{K}$ le compact $[-\infty,+\infty]^{\omega}$, muni de la topologie produit de celle de $[-\infty,+\infty]$. On prolonge à $\mathbb{K}$ le préordre de $\mathbb{R}^{\omega}$ en convenant que, pour tout $t \in \mathbb{R},|-\infty|=|+\infty|=+\infty>|t|$; donc dans $\mathbb{K}$,

$$
x \leq_{\omega} y \Leftrightarrow \forall k,|x(k)| \leq|y(k)| .
$$

On prolonge à $\mathbb{K}$ la notion d'hérédité, et si $A \subseteq \mathbb{K}$, on notera $\operatorname{Her}(A)$ la plus petite partie héréditaire contenant $A$ :

$$
\operatorname{Her}(A)=\left\{x \mid \exists y \in A, x \leq_{\omega} y\right\} .
$$

On notera par l'indice ou l'exposant " $\mathbb{K}$ " les notions relatives à $\mathbb{K}$ et à sa topologie.

On va maintenant étudier brièvement le rapport entre topologie et préordre dans $\mathbb{K}$.

Propriété (3.4.4). (i) Il y a une injection de l'espace $\mathcal{F}\left(\mathbb{R}^{\omega}\right)$ des fermés de $\mathbb{R}^{\omega}$ dans l'espace $\mathcal{K}(\mathbb{K})$ des compacts de $\mathbb{K}$. Cette injection est $F \mapsto \bar{F}^{\mathbb{K}}$, et un inverse à gauche est $K \mapsto K \cap \mathbb{R}^{\omega}$. De plus $F \in \mathcal{F}\left(\mathbb{R}^{\omega}\right)$ est héréditaire si et seulement si $\bar{F}^{\mathbb{K}}$ l'est.

(ii) Si $K \in \mathcal{K}(\mathbb{K})$, alors $\operatorname{Her}(K) \in \mathcal{K}(\mathbb{K})$; de plus $K \mapsto \operatorname{Her}(K)$ est continue sur $\mathcal{K}(\mathbb{K})$ et l'ensemble des fermés héréditaires est un compact de $\mathcal{K}(\mathbb{K})$.

Preuve. (i) Le premier fait (propriétés de l'injection décrite) est classique et sert en général à prouver que $\mathcal{F}\left(\mathbb{R}^{\omega}\right)$ est un borélien standard.

Si $F$ est héréditaire et $x \in \operatorname{Her}\left(\bar{F}^{\mathbb{K}}\right)$, on a $y \in \bar{F}^{\mathbb{K}}$ tel que $x \leq_{\omega} y ; y$ est limite dans $\mathbb{K}$ d'une suite $\left(z_{n}\right)_{n}$ de points de $F$, mais alors on vérifie aisément que $x \wedge z_{n}$ tend vers $x$, et comme $x \wedge z_{n} \leq_{\omega} z_{n} \in F$, et que $F$ est héréditaire, $x$ est limite d'une suite de points de $F$, donc est dans $\bar{F}^{\mathbb{K}}$. Par suite $\bar{F}^{\mathbb{K}} \supseteq \operatorname{Her}\left(\bar{F}^{\mathbb{K}}\right)$, d'où $\bar{F}^{\mathbb{K}}=\operatorname{Her}\left(\bar{F}^{\mathbb{K}}\right)$ et $\bar{F}^{\mathbb{K}}$ est bien héréditaire.

Réciproquement, si $\bar{F}^{\mathbb{K}}$ est héréditaire, $F=\bar{F}^{\mathbb{K}} \cap \mathbb{R}^{\omega}$ l'est aussi, comme intersection de deux héréditaires.

(ii) Supposons que $K \in \mathcal{K}(\mathbb{K})$, et montrons que $\operatorname{Her}(K)$ est fermé dans $\mathbb{K}$.

Soit une suite $\left(z_{n}\right)_{n}$ de points de $\operatorname{Her}(K)$ convergeant vers $z \in \mathbb{K}$. On a donc pour chaque $n$ un point $x_{n}$ dans $K$ tel que $z_{n} \leq_{\omega} x_{n}$; comme $K$ est compact, il y a une sous-suite convergente $\left(x_{n_{k}}\right)_{k}$, avec une limite $x \in K$. Or on a $z_{n_{k}} \leq_{\omega} x_{n_{k}}$ et $z_{n_{k}}$ tend vers $z$, donc $z \leq_{\omega} x \in K$ et $z \in \operatorname{Her}(K)$, ce qui prouve bien que $\operatorname{Her}(K)$ est fermé.

Montrons maintenant que l'application $K \mapsto \operatorname{Her}(K)$ est continue de $\mathcal{K}(\mathbb{K})$ dans lui-même. 
Il suffit de vérifier que l'image d'une suite convergente de compacts est une suite de compacts qui converge vers l'image de la limite. On rappelle que $\lim _{n \rightarrow+\infty} K_{n}=K$ si et seulement si

$$
\forall x \in K, \exists\left(x_{n}\right)_{n}, \quad\left(\forall n, x_{n} \in K_{n}\right) \text { et }\left(\lim _{n \rightarrow+\infty} x_{n}=x\right)
$$

et

$$
\forall x, \quad\left(\exists\left(x_{n}\right)_{n}, \forall n, x_{n} \in K_{n} \text { et } \exists\left(n_{i}\right)_{i}, \lim _{i \rightarrow+\infty} x_{n_{i}}=x\right) \Rightarrow x \in K .
$$

Considérons donc une suite $\left(K_{n}\right)_{n}$ de compacts qui converge vers $K$. Montrons qu'on a $\lim _{n \rightarrow+\infty} \operatorname{Her}\left(K_{n}\right)=\operatorname{Her}(K)$.

Soit $x \in \operatorname{Her}(K)$. On a donc un $y \in K$ tel que $x \leq_{\omega} y$; et aussi, par propriété de la limite, une suite $\left(y_{n}\right)_{n}$ avec $y_{n} \in K_{n}$ et $\lim _{n \rightarrow+\infty} y_{n}=y$. Si on pose, pour tous $k, n$,

$$
x_{n}(k)=\operatorname{sgn}(x(k)) \inf \left(|x(k)|,\left|y_{n}(k)\right|\right),
$$

on a alors clairement $x_{n} \leq_{\omega} y_{n}$, donc $x_{n} \in \operatorname{Her}\left(K_{n}\right)$ et puisque $x \leq_{\omega} y=$ $\lim _{n \rightarrow+\infty} y_{n}$ il s'ensuit que

$$
\forall k, \quad|x(k)| \leq|y(k)|=\lim _{n \rightarrow+\infty}\left|y_{n}(k)\right|
$$

et par conséquent

$$
\lim _{n \rightarrow+\infty} \inf \left(\left|y_{n}(k)\right|,|x(k)|\right)=|x(k)| .
$$

Finalement, $\lim _{n \rightarrow+\infty} x_{n}=x$ avec $x_{n} \in \operatorname{Her}\left(K_{n}\right)$, donc la première condition pour que $\operatorname{Her}(K)$ soit la limite de la suite $\left(\operatorname{Her}\left(K_{n}\right)\right)_{n}$ est vérifiée.

Soient maintenant $x_{n} \in \operatorname{Her}\left(K_{n}\right)$ et $\left(n_{i}\right)_{i}$ tels que $\lim _{i \rightarrow+\infty} x_{n_{i}}=x$; montrons que $x \in \operatorname{Her}(K)$.

De $x_{n} \in \operatorname{Her}\left(K_{n}\right)$ on tire qu'il existe des $y_{n} \in K_{n}$ tels que $x_{n} \leq_{\omega} y_{n}$; par compacité de $\mathbb{K}$ on peut extraire de $\left(y_{n_{i}}\right)_{i}$ une sous-suite $\left(y_{n_{i(k)}}\right)_{k}$ convergeant vers un $y$, et $y \in K$ puisque $K$ est la limite des $K_{n}$. Comme $x_{n_{i(k)}} \leq_{\omega} y_{n_{i(k)}}$ on aura $x \leq_{\omega} y \in K$, et on a bien $x \in \operatorname{Her}(K)$.

Ainsi $K \mapsto \operatorname{Her}(K)$ est bien continue, et l'image du compact $\mathcal{K}(\mathbb{K})$, c'està-dire l'ensemble des compacts héréditaires de $\mathcal{K}(\mathbb{K})$, est bien un compact.

DÉfinition (3.4.5). Dans $\mathcal{K}(\mathbb{K})$ on définit les ensembles suivants :

- $\mathcal{H}$ est l'ensemble des compacts héréditaires ;

- $\mathcal{P}$ est l'ensemble des compacts héréditaires $K$ tels que $K \cap \mathbb{R}^{\omega}$ soit un fermé héréditaire petit;

- $\mathcal{G}$ est l'ensemble des compacts héréditaires $K$ tels que $K \cap \mathbb{R}^{\omega}$ soit un fermé héréditaire grand;

- $\Psi$ est l'ensemble des compacts $K$ pour lesquels il y a un $x \in X$ tel que $\operatorname{Her}(K) \cap C[x]$ est rare dans $C[x]$. 
Traduisons tout de suite, avec ces définitions, les propriétés des ensembles petits et grands en tenant compte de (3.4.1) :

PropriÉté (3.4.6). Sous (3.4.1) on $a$ :

- $\mathcal{P}=\mathcal{H} \cap \Psi$;

- $\Psi$ est un $\sigma$-idéal;

- si $K \in \mathcal{H}$, alors $K \in \mathcal{G}$ si et seulement si pour tout $x \in X$, il y a $n>0$ entier tel que $n^{-1} \pi_{n}(x)+\varepsilon_{n}(x) \in K$.

Preuve. La seule propriété vraiment nouvelle est le caractère $\sigma$-idéal de $\Psi$, mais c'est une conséquence de (3.4.1) et de $\operatorname{Her}\left(\bigcup_{\iota} A_{\iota}\right)=\bigcup_{\iota} \operatorname{Her}\left(A_{\iota}\right)$ pour toute famille $\left\{A_{\iota}\right\}_{\iota}$.

On va maintenant préciser la complexité de $\Psi$.

Propriété (3.4.7). $\Psi$ et $\mathcal{P}$ sont des $\mathrm{G}_{\delta}$ de $\mathcal{K}(\mathbb{K}) ; \mathcal{G}$ est un $\mathrm{F}_{\sigma}$.

Preuve. D'après le théorème Kechris-Louveau-Woodin [K-L-W] sur les $\sigma$-idéaux de compacts, il suffit de montrer que $\Psi$ est analytique pour prouver qu'il est $\mathrm{G}_{\delta} ; \mathcal{P}=\mathcal{H} \cap \Psi$ le sera aussi d'après (3.4.4), et $\mathcal{G}=\mathcal{H}-\Psi$ sera $\mathrm{F}_{\sigma}$.

On a

$$
K \in \Psi \Leftrightarrow \exists x \in X, \operatorname{Her}(K) \cap C[x] \text { est rare dans } C[x] .
$$

Or

$$
\begin{aligned}
K & \cap C[x] \text { est rare dans } C[x] \\
& \Leftrightarrow \forall N, \forall A \in \mathbb{Q}^{N} \cap C[x],\left(A+W_{N}\right) \cap C[x] \nsubseteq K \\
& \Leftrightarrow \forall N, \forall A \in \mathbb{Q}^{N} \cap C[x], \exists y, y \in\left(A+W_{N}\right) \text { et } y \leq_{\omega} x \text { et } y \notin K .
\end{aligned}
$$

Pour chaque $N, A \in \mathbb{Q}^{N}$, l'ensemble

$$
\left\{(x, K) \mid A \not_{\omega} x \text { ou }\left(\exists y, y \in\left(A+W_{N}\right) \text { et } y \leq_{\omega} x \text { et } y \notin K\right)\right\}
$$

est analytique, donc l'intersection $E$ pour tous les couples $(N, A)$ l'est aussi. Or $\Psi=\{K \mid \exists x \in X,(x, \operatorname{Her}(K)) \in E\}, X$ est supposé analytique et $K \mapsto \operatorname{Her}(K)$ est continue. Donc $\Psi$ est analytique.

On va pouvoir tirer de ce fait une conséquence importante : on peut mettre en évidence une suite "fondamentale" de fermés héréditaires grands.

Proposition (3.4.8). Sous (3.4.1), il y a une famille dénombrable de fermés héréditaires grands $\left\{F_{n}\right\}_{n}$ tels que pour tout fermé héréditaire grand $F$, il $y$ ait $n$ tel que $F_{n} \subseteq F$.

Preuve. Une première réduction aisée est qu'il suffit de montrer qu'il existe des $K_{n} \in \mathcal{G}$ tels que pour tout $K \in \mathcal{G}$, il y ait $n$ tel que $K_{n} \subseteq K$.

Une deuxième réduction est qu'il suffit de montrer que si $\Phi \subseteq \mathcal{G}$ est fermé dans $\mathcal{K}(\mathbb{K})$, alors il y a une famille $K_{n} \in \Phi$ qui satisfait la même propriété 
restreinte à $\Phi$. En effet, c'est suffisant car $\mathcal{G}$ lui-même est $\mathrm{F}_{\sigma}$ de $\mathcal{K}(\mathbb{K})$ par (3.4.7), $\mathcal{G}=\bigcup_{p} \mathcal{G}_{p}$, donc il suffira de prendre la réunion des familles trouvées pour chaque $\mathcal{G}_{p}$.

Soit donc $\Phi \subseteq \mathcal{G}$ un fermé non vide de $\mathcal{K}(\mathbb{K})$, qui est donc compact métrisable pour la topologie induite par celle de $\mathcal{K}(\mathbb{K})$.

LEMME (3.4.9). Il y a un ouvert relatif $U \subseteq \Phi$ tel que $\bigcap\{K \mid K \in U\} \in \mathcal{G}$.

Preuve. Considérons une base dénombrable d'ouverts relatifs de $\Phi$, notée $\left\{U_{n}\right\}_{n}$. Posons $\Gamma=\bigcup_{n} \bigcap\left\{K \mid K \in U_{n}\right\}$. Alors pour tout $x \in X$, $\Gamma \cap C[x]$ n'est pas maigre dans $C[x]$. En effet, si $K \in \Phi$, par (3.4.6), on a $m>0$ tel que $m^{-1} \pi_{m}(x)+\varepsilon_{m}(x) \in K$. Donc si on pose

$$
\Phi_{m}=\left\{K \in \Phi \mid m^{-1} \pi_{m}(x)+\varepsilon_{m}(x) \in K\right\},
$$

on voit que les $\Phi_{m}$ sont des fermés de $\Phi$ dont la réunion est $\Phi$. $\Phi$ est compact (fermé de $\mathcal{K}(\mathbb{K})$ ), donc de Baire, il y a donc un des $\Phi_{m}$ dont l'intérieur est non vide, il y a donc $m, n$ tels que $U_{n} \subseteq \Phi_{m}$.

Cela veut dire que pour tout $K \in U_{n}$, on aura $m^{-1} \pi_{m}(x)+\varepsilon_{m}(x) \in K$; d'où $m^{-1} \pi_{m}(x)+\varepsilon_{m}(x) \in \bigcap\left\{K \mid K \in U_{n}\right\}$. Comme ce dernier ensemble est héréditaire, on a

$$
C\left[m^{-1} \pi_{m}(x)+\varepsilon_{m}(x)\right] \subseteq \bigcap\left\{K \mid K \in U_{n}\right\} \subseteq \Gamma,
$$

et $\Gamma \cap C[x]$ n'est effectivement pas maigre dans $C[x]$.

Donc $\Gamma \cap \mathbb{R}^{\omega}=\bigcup_{n} \cap\left(\left\{K \mid K \in U_{n}\right\} \cap \mathbb{R}^{\omega}\right)$ est un ensemble héréditaire grand, et par (3.4.1) pour un certain $n, \bigcap\left\{K \mid K \in U_{n}\right\} \cap \mathbb{R}^{\omega}$ est grand.

Or $\bigcap\left\{K \mid K \in U_{n}\right\}$ est compact héréditaire, donc cela signifie bien qu'il appartient à $\mathcal{G}$ et cela conclut la preuve du lemme : $U=U_{n}$ convient.

Fin de la preuve de (3.4.8). On peut construire de proche en proche une suite transfinie $\Phi^{\alpha}$ de fermés inclus dans $\Phi$ comme suit :

- $\Phi^{0}=\Phi$;

- si $\Phi^{\alpha} \neq \emptyset$, on applique (3.4.9) à $\Phi^{\alpha}$ : il y a un ouvert relatif $U^{\alpha}$ de $\Phi^{\alpha}$ tel que $\bigcap\left\{K \mid K \in U^{\alpha}\right\}$ est dans $\mathcal{G}$; on pose $\Phi^{\alpha+1}=\Phi^{\alpha}-U^{\alpha}$;

- si $\alpha$ est limite, $\Phi^{\alpha}=\bigcap_{\lambda<\alpha} \Phi^{\lambda}$.

Une telle suite est forcément de longueur dénombrable, il y a donc un premier $\gamma<\omega_{1}$ tel que $\Phi^{\gamma}=\emptyset$. Prenons pour $K_{n}$ une énumération des ensembles $\bigcap\left\{K \mid K \in U^{\alpha}\right\}$ pour tous les $\alpha<\gamma$.

Comme $\Phi=\bigcup_{\alpha<\gamma} U^{\alpha}$, on a pour tout $L \in \Phi$ un $\alpha<\gamma$ tel que $L \in U^{\alpha}$, d'où un $n$ tel que $K_{n}=\bigcap\left\{K \mid K \in U^{\alpha}\right\} \subseteq L$. Donc les $K_{n}$ conviennent bien.

On va maintenant préciser un certain nombre de propriétés possibles de ces "suites fondamentales" de compacts. En fait, on aura besoin de celles contenues dans la définition suivante : 
DÉfinition (3.4.10). On appelle bonne suite de compacts de $\mathbb{K}$ une suite $\left(K_{n}\right)_{n}$ de compacts vérifiant :

(a) pour tout $n, K_{n}$ est un compact héréditaire tel que $K_{n} \cap \mathbb{R}^{\omega}=F_{n}$ soit un fermé héréditaire grand;

(b) $K_{0}=\mathbb{K}$ et la suite est décroissante : pour tout $n, K_{n} \supseteq K_{n+1}$;

(c) pour tout $n$, on a $F_{n+1}+F_{n+1}+F_{n+1} \subseteq K_{n}$, c'est-à-dire que

$$
x, y, z \in K_{n+1} \cap \mathbb{R}^{\omega} \Rightarrow x+y+z \in K_{n} ;
$$

(d) pour tout compact $K$ de $\mathbb{K}$ héréditaire tel que $K \cap \mathbb{R}^{\omega}$ soit un fermé grand, il y a $n$ tel que $K_{n} \subseteq K$;

(e) $\bigcap_{n} K_{n}=\{0\}$;

(f) pour tout $x \in \mathbb{K}$,

$$
x \in X \Leftrightarrow \forall n, \exists m>0, m^{-1} \pi_{m}(x)+\varepsilon_{m}(x) \in K_{n},
$$

en convenant que $m^{-1} \cdot(+\infty)=+\infty$ et $m^{-1} \cdot(-\infty)=-\infty$.

On va maintenant prouver qu'il existe une bonne suite; (3.4.8) nous assure déjà qu'il existe des suites de compacts vérifiant (d).

Propriété (3.4.11). La propriété (d) de (3.4.10) entraîne (e) et (f).

Preuve. Soit

$$
\begin{aligned}
\bar{W}_{N}^{\mathbb{K}} & =\left\{x \in \mathbb{K}|\forall k<N,| x(k) \mid \leq \frac{1}{N+1}\right\} \\
& =\left[-\frac{1}{N+1}, \frac{1}{N+1}\right]^{N} \times[-\infty,+\infty]^{\omega-N} .
\end{aligned}
$$

On a $\bar{W}_{N}^{\mathbb{K}} \in \mathcal{G}$, donc $\bigcap_{n} K_{n} \subseteq \bigcap_{N} \bar{W}_{N}^{\mathbb{K}}=\{0\}$. Ceci prouve aussi que si

$$
\forall n, \exists m>0, \quad m^{-1} \pi_{m}(x)+\varepsilon_{m}(x) \in K_{n},
$$

alors

$$
\forall N, \exists m>0, \quad m^{-1} \pi_{m}(x)+\varepsilon_{m}(x) \in \bar{W}_{N}^{\mathbb{K}},
$$

et pour tout $N, \pi_{N}(x) \in \mathbb{R}^{\omega}$, c'est-à-dire que $x \in \mathbb{R}^{\omega}$

Maintenant si $x \in X$, il vérifie $\forall n, \exists m>0, m^{-1} \pi_{m}(x)+\varepsilon_{m}(x) \in K_{n}$ par (3.2.3) (en particulier on a bien $0 \in \bigcap_{n} K_{n}$ ).

Si $x \in \mathbb{R}^{\omega}-X$, montrons

$$
\exists n, \forall m>0, \quad m^{-1} \pi_{m}(x)+\varepsilon_{m}(x) \notin K_{n},
$$

ou ce qui revient au même d'après $(d)$,

$$
\exists K \in \mathcal{G}, \forall m>0, \quad m^{-1} \pi_{m}(x)+\varepsilon_{m}(x) \notin K .
$$

Considérons l'idéal $\mathcal{I}$ des parties $A$ de $\omega$ telles que $\pi_{A}(x) \in X$. C'est un idéal analytique comme $X$, libre et non trivial. On peut donc utiliser le 
théorème de Talagrand $[\mathrm{T}]$ : il y a une partition de $\omega$ en parties finies $F_{p}$ telle que pour $B \subseteq \omega, B$ est fini $\Leftrightarrow \bigcup_{p \in B} F_{p} \in \mathcal{I}$.

On n'a notamment qu'un nombre fini de $F_{p}$ tels que $x(k)=0$ pour tout $k \in F_{p}$. On peut supposer qu'il n'y en a aucun, quitte à répartir leurs éléments dans les autres $F_{p}$, ce qui ne change rien.

Considérons le compact suivant :

$$
K=\left\{t \in \mathbb{K} \mid \forall p, \exists k \in F_{p}, \quad\left(x(k) \neq 0 \text { et }|t(k)| \leq \frac{1}{2}|x(k)|\right)\right\} .
$$

C'est clairement un compact héréditaire.

Soit $t \in X$; posons $A=\left\{k|| t(k)\left|>\frac{1}{2}\right| x(k) \mid\right.$ ou $\left.x(k)=0\right\}$. On a $\pi_{A}(x) \leq_{\omega} 2 \pi_{A}(t)$, donc $A \in \mathcal{I}$. Cet ensemble ne contient qu'un nombre fini de $F_{p}$, sinon il y aurait une réunion $C$ d'une infinité qui serait élément de $\mathcal{I}$, ce qui est impossible.

Il y a donc un $p_{0}$ tel que

$$
\forall p \geq p_{0}, \exists k \in F_{p}, \quad|t(k)| \leq \frac{1}{2}|x(k)| \neq 0 .
$$

Soit $M$ le plus grand des éléments des $F_{p}$ pour $p<p_{0}$, et pour ces $p$ un $k_{p} \in F_{p}$ tel que $x\left(k_{p}\right) \neq 0$. Il y a un $m>M$ tel que $m^{-1}\left|t\left(k_{p}\right)\right| \leq \frac{1}{2}\left|x\left(k_{p}\right)\right|$ pour chacun des $p<p_{0}$. Si $t^{\prime}=m^{-1} \pi_{m}(t)+\varepsilon_{m}(t)$, on aura alors

$$
\forall p, \exists k \in F_{p}, \quad\left(x(k) \neq 0 \text { et }\left|t^{\prime}(k)\right| \leq \frac{1}{2}|x(k)|\right),
$$

donc $t^{\prime} \in K$. Ceci prouve bien que $K \in \mathcal{G}$.

Enfin pour tout $m$ il y a un $p$ tel que $F_{p} \cap m=\emptyset$; si $x^{\prime}=m^{-1} \pi_{m}(x)+$ $\varepsilon_{m}(x)$, on aura donc

$$
\forall k \in F_{p}, \quad\left(x(k)=0 \text { ou }\left|x^{\prime}(k)\right|=|x(k)|>\frac{1}{2}|x(k)|\right),
$$

ce qui montre que $x^{\prime} \notin K$, c'est-à-dire que $m^{-1} \pi_{m}(x)+\varepsilon_{m}(x) \notin K$ pour tout $m>0$, ce qui achève la démonstration.

On va maintenant prouver l'existence de bonnes suites de compacts.

Proprí́té (3.4.12). Il existe une bonne suite de compacts.

Preuve. On part d'une suite $\left(I_{p}\right)_{p}$ fournie par (3.4.8). Cette suite vérifie (d) par (3.4.8) et aussi (a), (e), (f) comme on l'a vu.

Posons $J_{p}=I_{0} \cap \ldots \cap I_{p}$ pour tout $p \in \omega$. Les $J_{p}$ sont toujours compacts et héréditaires et décroissent. Pour montrer que cette suite vérifie (a) il suffit de montrer qu'une intersection finie de fermés grands est grande.

Lemme (3.4.13). L'intersection de deux fermés grands de $\mathbb{R}^{\omega}$ est un fermé grand.

Preuve. Si $F, F^{\prime}$ sont deux tels fermés, $F \cap F^{\prime}$ est encore un fermé héréditaire, et si $x \in X$, par (3.2.3) on a

$$
\begin{aligned}
& \exists m>0, \quad m^{-1} \pi_{m}(x)+\varepsilon_{m}(x) \in F, \\
& \exists m^{\prime}>0, \quad m^{\prime-1} \pi_{m^{\prime}}(x)+\varepsilon_{m^{\prime}}(x) \in F^{\prime} .
\end{aligned}
$$


Si $M=\max \left(m, m^{\prime}\right)$, on a

$\frac{1}{M} \pi_{M}(x)+\varepsilon_{M}(x) \leq_{\omega} \frac{1}{m} \pi_{m}(x)+\varepsilon_{m}(x) \wedge \frac{1}{m^{\prime}} \pi_{m^{\prime}}(x)+\varepsilon_{m^{\prime}}(x) \in F \cap F^{\prime}$.

Par (3.2.3), $F \cap F^{\prime}$ est grand.

Suite de la preuve de (3.4.12). La suite $\left(J_{p}\right)_{p}$ vérifie donc la décroissance, (a) et (d). On peut supposer qu'elle vérifie (b), quitte à décaler les indices pour rajouter $J_{0}=\mathbb{K}$.

Reste à montrer qu'on peut encore la modifier pour avoir (c).

Notons que toute suite $K_{n}=J_{p_{n}}$ extraite avec $\left(p_{n}\right)_{n}$ strictement croissante vérifie toujours (a) et (b), mais aussi (d) : en effet, si $K \in \mathcal{G}$, il y a $p$ tel que $J_{p} \subseteq K$ et $n$ tel que $p_{n} \geq p$, donc $K_{n}=J_{p_{n}} \subseteq K$.

Il suffit donc de montrer qu'on peut trouver $\left(J_{p}^{\prime}\right)_{p}$ telle que pour tout $p$ on ait

$$
\left(J_{p+1}^{\prime} \cap \mathbb{R}^{\omega}\right)+\left(J_{p+1}^{\prime} \cap \mathbb{R}^{\omega}\right) \subseteq J_{p}^{\prime},
$$

car la suite $\left(J_{2 p}^{\prime}\right)_{p}$ conviendra alors. Et pour pouvoir extraire de $\left(J_{p}\right)_{p}$ une telle suite, tout se ramène à la propriété suivante :

Pour tout $K \in \mathcal{G}$, on a $p$ tel que $\left(J_{p} \cap \mathbb{R}^{\omega}\right)+\left(J_{p} \cap \mathbb{R}^{\omega}\right) \subseteq K$.

On peut encore changer cette condition de la manière que voici (qui a un sens car $(x, y) \mapsto x \vee y$ est continue de $\mathbb{K}^{2}$ dans $\mathbb{K}$, et donc envoie des compacts sur un compact, etc.) :

Pour tout $K \in \mathcal{G}$, il y a $K^{\prime} \in \mathcal{G}$ tel que $\left(K^{\prime} \cap \mathbb{R}^{\omega}\right) \vee\left(K^{\prime} \cap \mathbb{R}^{\omega}\right) \subseteq K$.

Supposons ceci vrai, et concluons (3.4.12) : pour $x, y \in \mathbb{R}^{\omega}$, on a clairement $x+y \leq_{\omega} 2(x \vee y)$. Or si $K \in \mathcal{G}$, on a aussi $\frac{1}{2} K \in \mathcal{G}$ car $\frac{1}{2} K$ est un compact héréditaire et que si $x \in X$, on a $2 x \in X$, donc par (3.2.3) il y a $m$ tel que $m^{-1} \pi_{m}(2 x)+\varepsilon_{m}(2 x) \in K$ et donc $m^{-1} \pi_{m}(x)+\varepsilon_{m}(x) \in \frac{1}{2} K$, ce qui par (3.2.3) prouve que $\frac{1}{2} K \in \mathcal{G}$. Si on peut trouver $K^{\prime} \in \mathcal{G}$ tel que $K^{\prime} \vee K^{\prime} \subseteq \frac{1}{2} K$, alors on aura bien $\left(K^{\prime} \cap \mathbb{R}^{\omega}\right)+\left(K^{\prime} \cap \mathbb{R}^{\omega}\right) \subseteq 2\left(K^{\prime} \vee K^{\prime}\right) \subseteq K$.

Pour conclure la preuve de (3.4.12), tout se ramène donc à :

Lemme (3.4.14). Pour tout $K \in \mathcal{G}$, il y a $K^{\prime} \in \mathcal{G}$ tel que $K^{\prime} \vee K^{\prime} \subseteq K$.

Preuve. Soit $K \in \mathcal{G}$; pour $n>0$ posons

$$
A_{n}=\left\{(x, y) \in \mathbb{K}^{2} \mid n^{-1} \pi_{n}(x \vee y)+\varepsilon_{n}(x \vee y) \in K\right\},
$$

qui est compact dans $\mathbb{K}^{2}$. Par hérédité de $K$ on a :

(i) $A_{n} \subseteq A_{n+1}$;

(ii) $\left(A_{n}\right)_{x}=\left\{y \mid(x, y) \in A_{n}\right\}$ est héréditaire pour tout $x \in \mathbb{K}$;

(iii) $\left(A_{n}\right)_{y} \subseteq\left(A_{n}\right)_{x}$ si $x \leq_{\omega} y$.

Soit $B_{n, p}=\left\{x \mid J_{p} \subseteq\left(A_{n}\right)_{x}\right\} \subseteq \mathbb{K}$. Par (iii) cet ensemble est héréditaire. Comme $A_{n}$ est compact, $B_{n, p}$ l'est aussi : si $x_{k} \in B_{n, p}$ converge dans $\mathbb{K}$ vers un $x$ et si $y \in J_{p}$, par compacité de $A_{n}$ et comme $\left(x_{k}, y\right) \in A_{n}$ puisque 
$J_{p} \subseteq\left(A_{n}\right)_{x_{k}}$, on a $(x, y) \in A_{n}$. Comme ceci est vrai pour tout $y \in J_{p}$, on a bien $J_{p} \subseteq\left(A_{n}\right)_{x}$ et $x \in B_{n, p}$.

Soit $x \in X$; pour tout $y \in X$, on a $x \vee y \leq_{\omega}|x|+|y|$, donc $x \vee y \in X$ et par (3.2.3) il y a $n$ tel que $n^{-1} \pi_{n}(x \vee y)+\varepsilon_{n}(x \vee y) \in K$, c'est-à-dire $(x, y) \in A_{n}$.

Ainsi $X \subseteq \bigcup_{n}\left(A_{n}\right)_{x}$, ce qui prouve que cet ensemble, qui est héréditaire par (ii), a une trace grande sur $\mathbb{R}^{\omega}$. Donc l'un des $\left(A_{n}\right)_{x} \cap \mathbb{R}^{\omega}$ est grand par (3.4.1), donc par construction des $J_{p}$ il y a $p$ tel que $J_{p} \subseteq\left(A_{n}\right)_{x}$, c'est-à-dire $x \in B_{n, p}$.

On a donc $\bigcup_{n, p} B_{n, p} \supseteq X$, et cette union de compacts héréditaire dont la trace sur $\mathbb{R}^{\omega}$ contient $X$ et est donc grande, a forcément un de ses termes dans $\mathcal{G}$.

Il y a donc $n_{0}, p_{0}$ tels que que $B_{n_{0}, p_{0}} \in \mathcal{G}$. Posons $L=J_{p_{0}} \cap B_{n_{0}, p_{0}} \in \mathcal{G}$ par (3.4.13). On a $L \times L \subseteq B_{n_{0}, p_{0}} \times J_{p_{0}} \subseteq A_{n_{0}}$ par définition des $A_{n}, B_{n, p}$, et si on pose

$$
K^{\prime}=\left\{n_{0}^{-1} \pi_{n_{0}}(x)+\varepsilon_{n_{0}}(x) \mid x \in L\right\},
$$

on a bien là un compact de $\mathcal{G}$ tel que $K^{\prime} \vee K^{\prime} \subseteq K$.

On va maintenant pouvoir attaquer la construction essentielle de ce paragraphe : s'il y a une bonne suite de compacts, on peut construire une évaluation $\varphi$ sur $\mathbb{R}^{\omega}$ telle que $X=\operatorname{Exh}(\varphi)$.

Proposition (3.4.15). S'il y a une bonne suite de compacts pour $X$, alors il y a une évaluation $\varphi$ telle que $X=\operatorname{Exh}(\varphi)$. En particulier sous (3.4.1), X est polonisable comme espace vectoriel.

Preuve. Considérons donc notre bonne suite $\left(K_{n}\right)_{n}$ qui vérifie les conditions de (3.4.10)

On va commencer par définir des fonctions sur $c_{00}$. Pour tout $x \in c_{00}$ on pose

$$
\begin{aligned}
& \Psi_{0}(x)=\inf \left\{2^{-n} \mid x \in K_{n}\right\} \\
& \Psi_{1}(x)=\inf \left\{\sum_{1 \leq k \leq p} \Psi_{0}\left(x_{k}\right) \mid p \geq 1, x_{k} \in c_{00}, x \leq_{\omega} \sum_{1 \leq k \leq p} x_{k}\right\} .
\end{aligned}
$$

On a tout de suite le fait suivant :

Lemme (3.4.16). Pour tous $x, y \in c_{00}$, on $a$ :

- $0 \leq \frac{1}{2} \Psi_{0}(x) \leq \Psi_{1}(x) \leq \Psi_{0}(x) \leq 1$ et $\Psi_{0}(x)=0 \Leftrightarrow x=0$;

- $\Psi_{1}(x+y) \leq \Psi_{1}(x)+\Psi_{1}(y)$ et $x \leq_{\omega} y \Rightarrow \Psi_{1}(x) \leq \Psi_{1}(y)$

- pour tout $n, \Psi_{1}$ est continue sur $\mathbb{R}^{n}$.

Preuve. Voyons la deuxième assertion : $\Psi_{1}(x) \leq \Psi_{1}(y)$ est clair si $x \leq_{\omega} y$. Par ailleurs, en remarquant que l'hérédité des $K_{n}$ entraîne que $\Psi_{0}(t)=\Psi_{0}(|t|)$, on a pour tous $x_{k}, y_{k}$, 


$$
\begin{aligned}
x \leq_{\omega} \sum_{1 \leq k \leq p} x_{k} \text { et } y \leq_{\omega} \sum_{1 \leq k \leq p^{\prime}} y_{k} & \Rightarrow x \leq_{\omega} \sum_{1 \leq k \leq p}\left|x_{k}\right| \text { et } y \leq_{\omega} \sum_{1 \leq k \leq p^{\prime}}\left|y_{k}\right| \\
& \Rightarrow x+y \leq \omega \sum_{1 \leq k \leq p}\left|x_{k}\right|+\sum_{1 \leq k \leq p^{\prime}}\left|y_{k}\right|,
\end{aligned}
$$

et donc on voit aisément que $\Psi_{1}(x+y) \leq \Psi_{1}(x)+\Psi_{1}(y)$.

Voyons maintenant la première assertion : $0 \leq \Psi_{1}(x) \leq \Psi_{0}(x) \leq 1$ provient directement des définitions (et du fait que $K_{0}=\mathbb{K}$ ). De plus,

$$
x=0 \Leftrightarrow x \in \bigcap_{n} K_{n} \Leftrightarrow x \in \text { une infinité de } K_{n} \Leftrightarrow \Psi_{0}(x)=0 .
$$

Reste à montrer que $\Psi_{0}(x) \leq 2 \Psi_{1}(x)$, ou encore que

$$
x \leq_{\omega} \sum_{1 \leq k \leq p} x_{k} \Rightarrow \Psi_{0}(x) \leq 2 \sum_{1 \leq k \leq p} \Psi_{0}\left(x_{k}\right) .
$$

En fait, grâce à l'hérédité, tout se ramène à prouver que pour $p \geq 1$, et $x_{1}, \ldots, x_{p} \in c_{00}$, on a

$$
\Psi_{0}\left(\sum_{1 \leq k \leq p} x_{k}\right) \leq 2 \sum_{1 \leq k \leq p} \Psi_{0}\left(x_{k}\right) .
$$

On montre ceci par récurrence sur $p$. Si $p=1$, cela s'écrit $\Psi_{0}\left(x_{1}\right) \leq$ $2 \Psi_{0}\left(x_{1}\right)$ et il n'y a rien à prouver. Supposons que c'est vrai pour toute famille de $p$ suites finies, et montrons-le pour $x_{1}, \ldots, x_{p+1} \in c_{00}$.

Quitte à changer l'ordre on peut supposer que $\Psi_{0}\left(x_{1}\right) \leq \Psi_{0}\left(x_{k}\right)$ pour tout $k$, et que les $x_{k}$ sont non nuls sans quoi on est tout de suite ramené au cas de $p$ suites.

On a donc

$$
\Psi_{0}\left(x_{1}\right) \leq \frac{1}{2} \sum_{1 \leq k \leq p+1} \Psi_{0}\left(x_{k}\right),
$$

et il y a un premier $r \leq 1$ tel que

$$
\sum_{1 \leq k \leq r+1} \Psi_{0}\left(x_{k}\right)>\frac{1}{2} \sum_{1 \leq k \leq p+1} \Psi_{0}\left(x_{k}\right) .
$$

On aura donc par hypothèse de récurrence :

$$
\begin{aligned}
\Psi_{0}\left(\sum_{1 \leq k \leq r} x_{k}\right) & \leq 2 \sum_{1 \leq k \leq r} \Psi_{0}\left(x_{k}\right) \leq \sum_{1 \leq k \leq p+1} \Psi_{0}\left(x_{k}\right), \\
\Psi_{0}\left(\sum_{r+1<k \leq p+1} x_{k}\right) & \leq 2 \sum_{r+1<k \leq p+1} \Psi_{0}\left(x_{k}\right) \leq \sum_{1 \leq k \leq p+1} \Psi_{0}\left(x_{k}\right), \\
\Psi_{0}\left(x_{r+1}\right) & \leq \sum_{1 \leq k \leq p+1} \Psi_{0}\left(x_{k}\right) .
\end{aligned}
$$


Comme les $K_{n}$ forment une bonne suite de compacts, on a $x, y, z \in K_{n} \Rightarrow$ $x+y+z \in K_{n-1}$, d'où

$$
\Psi_{0}(x), \Psi_{0}(y), \Psi_{0}(z) \leq r \Rightarrow \Psi_{0}(x+y+z) \leq 2 r .
$$

Donc on a bien

$$
\Psi_{0}\left(\sum_{1 \leq k \leq p+1} x_{k}\right) \leq 2 \sum_{1 \leq k \leq p+1} \Psi_{0}\left(x_{k}\right)
$$

et la récurrence est achevée.

Reste la troisième assertion. Comme $\Psi_{1}$ est sous-additive, il suffit de prouver qu'elle est continue en 0 , c'est-à-dire que $\Psi_{1}(x)$ tend vers 0 quand $x$ tend vers 0 dans $\mathbb{R}^{n}$.

Comme $\Psi_{1} \leq \Psi_{0}$, il suffit de le montrer pour $\Psi_{0}$.

Or soit $p \in \omega$. $K_{p}$ est dans $\mathcal{G}$ et $\pi_{n}(I) \in c_{00} \in X$. Par (3.2.3) il y a $m>n$ tel que

$$
m^{-1} \pi_{m}\left(\pi_{n}(I)\right)+\varepsilon_{m}\left(\pi_{n}(I)\right)=m^{-1} \pi_{n}(I) \in K_{p},
$$

d'où par hérédité de $K_{p}$, dans $\mathbb{R}^{n}$,

$$
\|t\|_{\infty} \leq m^{-1} \Rightarrow t \in K_{p} \Rightarrow \Psi_{0}(t) \leq 2^{-p},
$$

ce qui prouve bien que $\lim _{t \in \mathbb{R}^{n}, t \rightarrow 0} \Psi_{0}(t)=0$.

Fin de la preuve de (3.4.15). On définit

$$
\forall x \in \mathbb{R}^{\omega}, \quad \varphi_{0}(x)=\sup \left\{\Psi_{1}\left(\pi_{n}(x)\right) \mid n \in \omega\right\} .
$$

Les propriétés $\mathrm{Ev}_{2}$ et $\mathrm{Ev}_{3}$ sont vérifiées puisqu'elles le sont pour $\Psi_{1}$. Ev 1 aussi : si $\varphi_{0}(x)=0$, c'est que $\Psi_{0}\left(\pi_{n}(x)\right) \leq 2 \Psi_{1}\left(\pi_{n}(x)\right) \leq \varphi_{0}(x)=0$. Donc pour tout $n, \pi_{n}(x)=0$, c'est-à-dire $x=0$.

On a $\mathrm{Ev}_{5}$ et $\mathrm{Ev}_{6}$, car chaque fonction $x \mapsto \pi_{n}(x) \mapsto \Psi_{1}\left(\pi_{n}(x)\right)$ est continue, et qu'une borne supérieure d'une famille de fonctions continues est semi-continue inférieurement, et même continue pour une famille finie de fonctions.

Reste $\mathrm{Ev}_{4}$ qui n'a aucune raison d'être vraie. Mais par $\mathrm{Ev}_{2}$ et $\mathrm{Ev}_{3}$, pour tout $x$ et tout $r \geq 1$ on a

$$
\varphi_{0}(r x) \leq[r] \varphi_{0}(x)+\varphi_{0}((r-[r]) x) \leq[r] \varphi_{0}(x)+\varphi_{0}(x) .
$$

Donc

$$
\frac{1}{r} \varphi_{0}(r x) \leq \frac{[r]}{r} \varphi_{0}(x)+\frac{1}{r} \varphi_{0}(x) \leq \varphi_{0}(x)+\varphi_{0}(x)=2 \varphi_{0}(x) .
$$

Si on pose $\varphi(x)=\sup \left\{r^{-1} \varphi_{0}(r x) \mid r \geq 1\right\}$, alors $\varphi$ vérifie encore $\mathrm{Ev}_{1}$, $\mathrm{Ev}_{2}, \mathrm{Ev}_{3}$, et aussi $\mathrm{Ev}_{4}$. Par ailleurs, étant une borne supérieure de fonctions semi-continues inférieurement, elle vérifie $\mathrm{Ev}_{5}$. Enfin, comme elle est sousadditive et majorée par $2 \varphi_{0}(x)$, on voit qu'elle est continue en 0 comme $\varphi_{0}$ et donc continue sur chaque $\mathbb{R}^{n}$. 
Reste à prouver que $X=\operatorname{Exh}(\varphi)$. Comme $\varphi_{0} \leq \varphi \leq 2 \varphi_{0}$, on a

$$
\lim _{n \rightarrow \infty} \varphi\left(\varepsilon_{n}(x)\right)=0 \Leftrightarrow \lim _{n \rightarrow \infty} \varphi_{0}\left(\varepsilon_{n}(x)\right)=0
$$

et donc tout revient à prouver que

$$
x \in X \Leftrightarrow \lim _{n \rightarrow \infty} \varphi_{0}\left(\varepsilon_{n}(x)\right)=0 .
$$

Si $x \in X$ et $k \in \omega$, il y a $N>0$ tel que $N^{-1} \pi_{N}(x)+\varepsilon_{N}(x) \in K_{k}$. Alors $\pi_{n}\left(\varepsilon_{N}(x)\right) \leq{ }_{\omega} N^{-1} \pi_{N}(x)+\varepsilon_{N}(x)$, donc par hérédité $\pi_{n}\left(\varepsilon_{N}(x)\right) \in K_{k}$ et $\Psi_{1}\left(\pi_{n}\left(\varepsilon_{N}(x)\right)\right) \leq \Psi_{0}\left(\pi_{n}\left(\varepsilon_{N}(x)\right)\right) \leq 2^{-k}$. Ceci étant vrai pour chaque $n$, $\varphi_{0}\left(\varepsilon_{N}(x)\right) \leq 2^{-k}$. Comme on peut trouver ce $N$ pour chaque $k$, on a bien $\varphi_{0}\left(\varepsilon_{N}(x)\right) \rightarrow 0$ et $x \in \operatorname{Exh}\left(\varphi_{0}\right)$.

Réciproquement, supposons $\lim _{n \rightarrow \infty} \varphi_{0}\left(\varepsilon_{n}(x)\right)=0$ et montrons que $x \in X$. Comme la suite des $K_{n}$ vérifie (f) de (3.4.10), il suffit de montrer

$$
\forall k, \exists m>0, \quad m^{-1} \pi_{m}(x)+\varepsilon_{m}(x) \in K_{k} .
$$

Soit donc $k \in \omega$. Comme $x \in \operatorname{Exh}\left(\varphi_{0}\right)$, on a un $n$ tel que $\varphi_{0}\left(\varepsilon_{n}(x)\right)<2^{-k-1}$. Comme $\varphi_{0}$ est continue sur $\mathbb{R}^{n}$, il y a un $m>n$ tel que

$$
\varphi_{0}\left(\varepsilon_{n}(x)\right)+\varphi_{0}\left(m^{-1} \pi_{n}(x)\right)<2^{-k-1} .
$$

D'où par la sous-additivité, et comme $m^{-1} \pi_{m}(x)+\varepsilon_{m}(x) \leq_{\omega} m^{-1} \pi_{n}(x)+$ $\varepsilon_{n}(x)$,

$$
\varphi_{0}\left(m^{-1} \pi_{m}(x)+\varepsilon_{m}(x)\right)<2^{-k-1} .
$$

Mais cela signifie que pour tout $l \in \omega$, on a

$$
\Psi_{1}\left(\pi_{l}\left(m^{-1} \pi_{m}(x)+\varepsilon_{m}(x)\right)\right) \leq 2^{-k-1} .
$$

Par (3.4.16) on a $\Psi_{0} \leq 2 \Psi_{1}$, donc pour tout $l$,

$$
\Psi_{0}\left(\pi_{l}\left(m^{-1} \pi_{m}(x)+\varepsilon_{m}(x)\right)\right) \leq 2^{-k},
$$

c'est-à-dire $\pi_{l}\left(m^{-1} \pi_{m}(x)+\varepsilon_{m}(x)\right) \in K_{k}$.

$K_{k}$ est compact dans $\mathbb{K}$, la suite $\pi_{l}\left(m^{-1} \pi_{m}(x)+\varepsilon_{m}(x)\right)$ converge vers $m^{-1} \pi_{m}(x)+\varepsilon_{m}(x)$ dans $\mathbb{R}^{\omega}$, donc dans $\mathbb{K}$, donc $m^{-1} \pi_{m}(x)+\varepsilon_{m}(x) \in K_{k}$.

Comme on peut trouver un tel $m$ pour chaque $k$, la preuve est achevée.

On peut donc exprimer la conclusion des paragraphes 3.3 et 3.4 dans le résultat suivant :

Proposition (3.4.17). Si $X$ est un sous-espace héréditaire de $\mathbb{R}^{\omega}$, analytique pour la topologie produit sur $\mathbb{R}^{\omega}$, alors on est exactement dans un des deux cas suivants:

(1) $X$ est polonisable comme espace vectoriel;

(2) on a $E_{1} \sqsubseteq_{\mathrm{c}} \equiv_{X}$, et même $c_{00} \leq_{\text {lin }}^{+} X$ ou $\ell_{\infty} \leq_{\text {lin }}^{+} X$. 


\section{CONSÉQUENCES POUR LES ESPACES POLONISABLES}

4.1. Topologie des espaces polonisables. Une des conséquences de la dichotomie précédente est qu'on connaît une classe de distances complètes canoniques définissant la topologie polonaise de tout espace héréditaire polonisable :

Proposition (4.1.1). Si $X$ est un espace analytique polonisable héréditaire, alors sa topologie polonaise est unique, et parmi les distances complètes qui l'engendrent il y en a au moins une engendrée par une évaluation $\varphi$ telle que $X=\operatorname{Exh}(\varphi)$.

Preuve. D'après la dichotomie, soit $X=\operatorname{Exh}(\varphi)$ pour une certaine évaluation $\varphi$, soit on peut plonger $E_{1}$ dans la relation quotient associée. Mais dans ce second cas, $X$ n'est pas du tout polonisable (cf. introduction), donc ce cas est exclu. On peut donc considérer $\varphi$, et alors tout revient à montrer que si $X$ est muni d'une autre distance $d$ complète satisfaisante, elle définit la même topologie. Mais c'est une conséquence aisée du théorème du graphe fermé : l'identité de $(X, \varphi)$ dans $(X, d)$ est linéaire et son graphe est fermée puisqu'il l'est dans la topologie produit, et que celle-ci est moins fine que les deux autres. Donc Id est continue, et dans le sens réciproque aussi pour les mêmes raisons. Donc les topologies sont équivalentes.

En fait, ce n'est pas la peine de supposer $X$ analytique : le seul fait d'être héréditaire et polonisable pour une topologie plus fine que la topologie produit suffit à assurer l'existence de $\varphi$. On en trouvera la preuve dans $[\mathrm{C}]$. On s'en tiendra néanmoins ici aux $X$ analytiques, ce qu'on ne répétera pas toujours, le sous-entendant.

Ainsi on connaît la forme de tous les espaces polonisables et on peut préciser certaines propriétés claires sur la forme $\operatorname{Exh}(\varphi)$. Notamment :

Proposition (4.1.2). Soit $X$ un sous-espace polonisable. Alors $X$ est $\Pi_{0}^{3}$ dans $\mathbb{R}^{\omega}$.

Preuve. En effet, il y a $\varphi$ telle que $X=\operatorname{Exh}(\varphi)$. Mais alors

$$
x \in X \Leftrightarrow \forall n>0, \exists m, \varphi\left(\varepsilon_{m}(x)\right) \leq 1 / n .
$$

Or la condition " $\varphi\left(\varepsilon_{m}(x)\right) \leq 1 / n$ " est fermée puisque $\varphi$ est sci, donc l'écriture précédente prouve bien que $X$ est $\boldsymbol{\Pi}_{0}^{3}$.

Pour préciser les classes exactes qu'on peut avoir, on va faire une étude plus précise.

4.2. Dichotomies pour les espaces polonisables. On va étudier un espace $E$ auquel est associé une évaluation $\varphi$ telle que $E=\operatorname{Exh}(\varphi)$. 
On se propose d'étudier si $\equiv_{E}$ domine, pour les hiérarchies définies plus haut, des relations fixes et si possible d'autres relations d'égalité modulo des espaces de $\mathbb{R}^{\omega}$.

Proposition (4.2.1). Soit $F$ un sous-espace héréditaire tel que $E \subseteq$ $F \subseteq \operatorname{Fin}(\varphi)$. Alors :

(1) si $F \neq \operatorname{Fin}(\varphi)$, et si $F$ est, vu comme partie de $\mathbb{R}^{\omega}$, dans la tribu $\sigma\left(\boldsymbol{\Sigma}_{1}^{1}\right)\left(\tau_{\omega}\right)$ engendrée par les analytiques de $\tau_{\omega}$, alors $c_{0} \leq_{\operatorname{lin}}^{+} F$ et $F$ est $\Pi_{3}^{0}$-hard; en particulier si $E \neq \operatorname{Fin}(\varphi)$,

$$
c_{0} \leq_{\operatorname{lin}}^{+} E \text {; }
$$

et $E$ est $\boldsymbol{\Pi}_{3}^{0}$-complet;

(2) si $F \neq E$, on a une injection $f$ linéaire continue $\leq_{\omega}$-croissante de $\mathbb{R}^{\omega}$ dans lui-même telle que $f^{-1}(F)=\ell_{\infty}$ et $f^{-1}(E)=c_{0}$; en particulier

$$
\ell_{\infty} \leq_{\operatorname{lin}}^{+} F
$$

et $F$ (par exemple $\operatorname{Fin}(\varphi)$ si $E \neq \operatorname{Fin}(\varphi))$ n'est pas polonisable comme groupe additif.

Preuve. (1) On a donc $x \in \operatorname{Fin}(\varphi)-F$. Considérons l'idéal $\mathcal{I}$ suivant : $A \in \mathcal{I} \Leftrightarrow \pi_{A}(x) \in F$. C'est clairement un idéal à cause des propriétés d'hérédité et algébriques de $F$; il est dans $\sigma\left(\boldsymbol{\Sigma}_{1}^{1}\right)$ car $F$ l'est dans $\mathbb{R}^{\omega}$ et que l'application $2^{\omega} \rightarrow \mathbb{R}^{\omega}, A \mapsto \pi_{A}(x)$, est continue, donc il est Baire-mesurable dans $2^{\omega}$.

On peut donc appliquer le théorème de Talagrand [T] à $\mathcal{I}$ : il y a une partition de $\omega$ en parties finies $\left\{F_{k}\right\}_{k}$ telle que pour $B \subseteq \omega$ on ait : $B$ est fini $\Leftrightarrow \bigcup_{k \in B} F_{k} \in \mathcal{I}$.

Considérons alors l'application suivante :

$$
f: \mathbb{R}^{\omega} \rightarrow \mathbb{R}^{\omega}, \quad t \mapsto f(t)=\sum_{k} t(k) \pi_{F_{k}}(x),
$$

qui est clairement linéaire continue et $\leq_{\omega}$-croissante, et injective si on suppose que pour tout $k$ on a $n \in F_{k}$ tel que $x(n) \neq 0$, ce qui n'est pas restrictif car les $F_{k}$ composés entièrement de $n$ tels que $x(n)=0$ ont pour réunion un ensemble de $\mathcal{I}$ et sont forcément en nombre fini, donc on peut toujours répartir les éléments de ces $F_{k}$ parmi les autres et supposer qu'il n'y a pas de tels $F_{k}$.

Maintenant si $t \in c_{0}$, soit $\varepsilon>0$; comme $x$ est dans $\operatorname{Fin}(\varphi)$, il y a $\eta>0$ tel que $|h| \leq \eta \Rightarrow \varphi(h x) \leq \varepsilon$, et il y a $K$ tel que $k \geq K \Rightarrow|t(k)| \leq \eta$.

Soit $N$ un entier plus grand que les éléments des $F_{k}$ pour $k<K$, ce qui est possible car ils sont finis.

Alors $\varepsilon_{N}(f(t))$ est nul sur les composantes qui, parmi les termes de la somme qui définit $f(t)$, viennent des termes en $t(k) \pi_{F_{k}}(x)$ pour les $k<K$; 
donc il reste des termes en $t(k) \pi_{F_{k}}(x)$ pour des $k \geq K$ et donc avec $|t(k)| \leq \eta$ et finalement

$$
\varepsilon_{N}(f(t)) \leq_{\omega} \eta \varepsilon_{N}(x) \leq_{\omega} \eta x,
$$

d'où l'on tire $\varphi\left(\varepsilon_{N}(f(t))\right) \leq \varphi(\eta x) \leq \varepsilon$.

On a donc montré

$$
\forall \varepsilon>0, \exists N, \quad \varphi\left(\varepsilon_{N}(f(t))\right) \leq \varepsilon, \quad \operatorname{donc} \quad f(t) \in \operatorname{Exh}(\varphi) \subseteq F .
$$

Soit $t \notin c_{0}$. Il y a donc un $r>0$ et une partie infinie $B \subseteq \omega$ tels que $k \in B \Rightarrow|t(k)| \geq r$. Alors si $C=\bigcup_{k \in B} F_{k}$, on a

$$
r \pi_{C}(x)=\sum_{k \in B} r \pi_{F_{k}}(x) \leq_{\omega} \sum_{k \in B} t(k) \pi_{F_{k}}(x) \leq_{\omega} f(t) ;
$$

or $C \notin \mathcal{I}$ car $B$ est infini, donc $\pi_{C}(x) \notin F$ et par hérédité $f(t) \notin F$.

Ainsi $f$ est bien un plongement de $c_{0}$ dans $F$. Les autres assertions en découlent puisque $c_{0}$ est $\Pi_{3}^{0}$-complet par (1.3.1) : si on plonge $c_{0}$ dans $F$, $F$ est bien $\Pi_{3}^{0}$-hard, et si c'est vrai pour $F=E$ qui est $\Pi_{3}^{0}$ par (4.1.2), cela veut bien dire que $E$ est $\Pi_{3}^{0}$-complet.

(2) Prenons $x \in F-E$; comme $x \notin E, \varphi\left(\varepsilon_{n}(x)\right)$ ne tend pas vers 0 ; cette suite décroissante est donc minorée strictement par un $a>0$.

Grâce à la semi-continuité de $\varphi$, si $n$ est donné,

$$
\varphi\left(\varepsilon_{n}(x)\right)>a
$$

donc il y a un $n^{\prime}>n$ tel que

$$
\varphi\left(\pi_{n^{\prime}}\left(\varepsilon_{n}(x)\right)\right)>a, \quad \text { c'est-à-dire } \varphi\left(\pi_{\left[n, n^{\prime}[\right.}(x)\right)>a .
$$

Il s'ensuit qu'on peut, de proche en proche, construire une suite $n_{0}=0<$ $n_{1}<n_{2}<\ldots$ telle que pour tout $k$,

$$
\varphi\left(\pi_{\left[n_{k}, n_{k+1}[\right.}(x)\right)>a .
$$

Considérons l'application suivante :

$$
f: \mathbb{R}^{\omega} \rightarrow \mathbb{R}^{\omega}, \quad(t(k))_{k} \mapsto \sum_{k} t(k) \pi_{\left[n_{k}, n_{k+1}[\right.}(x),
$$

qui est définie car une somme de suites à supports disjoints converge dans $\tau_{\omega}$, et clairement linéaire croissante pour $\leq_{\omega}$, et continue.

Comme $\varphi\left(\pi_{\left[n_{k}, n_{k+1}[\right.}(x)\right)>a$, on a $\pi_{\left[n_{k}, n_{k+1}[\right.}(x) \neq 0$ et (encore grâce aux supports disjoints) cela suffit à assurer $\operatorname{Ker}(f)=\{0\}$.

Maintenant soient $t \in c_{0}$ et $\varepsilon>0$. Comme $x \in \operatorname{Fin}(\varphi), \varphi(\lambda x)$ tend vers 0 avec $\lambda$ et il y a un $\eta>0$ tel que

$$
|\lambda|<\eta \Rightarrow \varphi(\lambda x)<\varepsilon .
$$


Comme $t \in c_{0}$ il y a $k_{0}$ tel que $k \geq k_{0} \Rightarrow|t(k)|<\eta / 2$. Alors pour $k \geq k_{0}$ et $n \in\left[n_{k}, n_{k+1}[\right.$, on a

$$
|t(k) x(n)| \leq \frac{\eta}{2}|x(n)|
$$

ce qui signifie bien que

$$
\varepsilon_{n_{k}}(f(t))=\sum_{k \geq k_{0}} t(k) \pi_{\left[n_{k}, n_{k+1}[\right.}(x) \leq_{\omega} \frac{\eta}{2} \sum_{n \geq n_{k_{0}}} x(n)=\frac{\eta}{2} \varepsilon_{n_{k}}(x) \leq_{\omega} \frac{\eta}{2} x .
$$

D'où $\varphi\left(\varepsilon_{n_{k}}(f(t))\right) \leq \varphi\left((\eta / 2) \varepsilon_{n_{k}}(x)\right)<\varepsilon$.

Comme $\varphi\left(\varepsilon_{n}(f(t))\right)$ décroît, elle doit tendre vers 0 et $f(t) \in \operatorname{Exh}(\varphi)=E$.

Soit $t \notin c_{0}$; il y a une suite de termes de $t,\left(t\left(k_{p}\right)\right)_{p}$, dont les valeurs absolues sont minorées par un $r>0$. Alors

$$
r \pi_{\left[n_{k_{p}}, n_{k_{p}+1}[\right.}(x) \leq_{\omega} t\left(k_{p}\right) \pi_{\left[n_{k_{p}}, n_{k_{p}+1}[\right.}(x)=\pi_{\left[n_{k_{p}}, n_{k_{p}+1}[\right.}(f(t)),
$$

donc

$$
\begin{aligned}
a & <\varphi\left(\pi_{\left[n_{k_{p}}, n_{k_{p}+1}[\right.}(x)\right)<\sup (1,1 / r) \varphi\left(r \pi_{\left[n_{k_{p}}, n_{k_{p}+1}[\right.}(x)\right) \\
& \leq \sup (1,1 / r) \varphi\left(\pi_{\left[n_{k_{p}}, n_{k_{p}+1}[\right.}(f(t))\right) \leq \sup (1,1 / r) \varphi\left(\varepsilon_{n_{k_{p}}}(f(t))\right)
\end{aligned}
$$

et finalement les $\varepsilon_{n_{k_{p}}}(f(t))$ sont minorés par $a / \sup (1,1 / r)>0$ et $\varepsilon_{n}(f(t))$ ne tend pas vers 0 , c'est-à-dire $f(t) \notin E$.

Finalement on a bien $f^{-1}(E)=c_{0}$.

Maintenant soit $t \in \ell_{\infty}$. On a clairement

$$
f(t)=\sum_{k} t(k) \pi_{\left[n_{k}, n_{k+1}[\right.}(x) \leq_{\omega} \sum_{k}\|t\|_{\infty} \pi_{\left[n_{k}, n_{k+1}[\right.}(x)=\|t\|_{\infty} x \in F,
$$

donc par hérédité $f(t) \in F$.

Si $t \notin \ell_{\infty}$, la suite des $|t(k)|$ est non majorée. Soit $\alpha>0$. Il y a sûrement un $k$ tel que $\alpha|t(k)| \geq 1$; alors

$$
\begin{aligned}
\varphi(\alpha f(t)) & \geq \varphi\left(\alpha \pi_{\left[n_{k_{p}}, n_{k_{p}+1}[\right.}(f(t))\right)=\varphi\left(\alpha t(k) \pi_{\left[n_{k_{p}}, n_{k_{p}+1}[\right.}(x)\right) \\
& \geq \varphi\left(\pi_{\left[n_{k_{p}}, n_{k_{p}+1}[\right.}(x)\right)>a .
\end{aligned}
$$

Ainsi $\varphi(\alpha f(t))$ ne tend pas vers 0 avec $\alpha$. D'où $f(t) \notin \operatorname{Fin}(\varphi)$ et a fortiori $f(t) \notin F$.

Le deuxième résultat signifie qu'on peut en fait trouver une copie isomorphe (pour la structure vectorielle, le préordre et la topologie) $X \subseteq \mathbb{R}^{\omega}$ de $\mathbb{R}^{\omega}$ tel que le préordre $\leq_{\omega}$ restreint à $X$ corresponde à l'image du préordre de $\mathbb{R}^{\omega}$ et que $X \cap E$ soit l'isomorphe de $c_{0}$, et $X \cap F$ l'isomorphe de $\ell_{\infty}$. C'est donc un plongement très "fidèle" qu'on a là.

Notons d'ailleurs qu'on n'a pas fait d'hypothèse dans la preuve de l'assertion (2) sur la complexité descriptive de $F$. 
On va s'intéresser maintenant au cas où $E=\operatorname{Fin}(\varphi)$. C'est bien sûr le cas des $\ell_{p}$ pour $p \neq \infty$ par exemple, mais il y a des exemples bien plus compliqués, notamment, on va le voir, qui sont $\boldsymbol{\Pi}_{3}^{0}$.

Prenons par exemple l'évaluation suivante (on vérifie aisément que c'en est une) :

$$
\varphi_{0}(x)=\sup _{k}\left\{|x(k)|^{1 / k}\right\} .
$$

Comme $\lim _{k}|t|^{1 / k}=1$ pour tout $t \neq 0$, on n'a $\lim _{t \rightarrow 0} \varphi_{0}(t x)=0$ que si $\varphi_{0}\left(\varepsilon_{k}(x)\right) \rightarrow 0$, c'est-à-dire $x \in \operatorname{Exh}\left(\varphi_{0}\right)$.

Donc $\operatorname{Fin}\left(\varphi_{0}\right)=\operatorname{Exh}\left(\varphi_{0}\right)$ mais on peut très bien avoir $\varphi_{0}(x)$ non infini et même aussi petit qu'on veut et $x \notin \operatorname{Fin}\left(\varphi_{0}\right)$.

On va voir que de tels espaces sont $\boldsymbol{\Pi}_{3}^{0}$-complets.

Proposition (4.2.2). Si $E=\operatorname{Fin}(\varphi) \neq \mathbb{R}^{\omega}$ alors on a exactement une des deux possibilités suivantes:

- E est un vrai $\boldsymbol{\Sigma}_{2}^{0}$; ou

- $E_{0}^{\omega} \sqsubseteq_{\mathrm{c}} \equiv_{E}$ et $E$ est un $\boldsymbol{\Pi}_{3}^{0}$-complet.

Preuve. $1^{\text {er }}$ CAS. Hors de $E, \varphi$ est minorée par un $a>0$. Alors si $x \in E$, on a $\varphi(t x) \rightarrow 0$, donc il y a $t$ tel que $\varphi(t x) \leq a / 2$.

Si $x \notin E$, on aura au contraire $\varphi(t x) \geq a>a / 2$ pour tout $t \neq 0$. D'où

$$
x \in E \Leftrightarrow \exists N, \varphi\left(\frac{1}{N+1} x\right) \leq a / 2,
$$

qui est bien une définition $\mathrm{F}_{\sigma}$ grâce à la semi-continuité inférieure de $\varphi$. C'est un vrai $\mathrm{F}_{\sigma}$ car on sait qu'un sous-groupe $\mathrm{G}_{\delta}$ d'un groupe polonais est nécessairement fermé et un ensemble fermé contenant $c_{00}$ est égal à l'adhérence ${\overline{c_{00}}}^{\omega}=\mathbb{R}^{\omega}$.

$2^{\text {ème }}$ CAS. Il y a des $x_{0}, x_{1}, \ldots$ tels que $x_{k} \notin E$ et $\varphi\left(x_{k}\right)<2^{-k}$. Alors pour tout $k$ on a un réel $\delta_{k}>0$ qui minore tous les $\varphi\left(\varepsilon_{n}\left(x_{k}\right)\right)$. On en déduit comme au (4.2.1) que pour tout $n$ il y a $n^{\prime}>n$ tel que $\varphi\left(\pi_{\left[n, n^{\prime}[\right.}\left(x_{k}\right)\right)>\delta_{k}$.

Construisons de proche en proche une suite $n_{0}=0<n_{1}<n_{2}<\ldots$ tels que si $k=\langle p, q\rangle$ alors $\varphi\left(\pi_{\left[n_{k}, n_{k+1}[\right.}\left(x_{p}\right)\right)>\delta_{p}$.

Considérons

$$
f: 2^{\omega^{2}} \rightarrow \mathbb{R}^{\omega}, \quad \alpha=(\alpha(p, q))_{p, q} \mapsto f(\alpha)=\sum_{p, q} \alpha(p, q) \pi_{\left[n_{\langle p, q\rangle}, n_{\langle p, q\rangle+1}[\right.}\left(x_{p}\right) .
$$

$f$ est bien définie et continue. De plus $|f(\alpha)-f(\beta)|=|f(\alpha \triangle \beta)|$.

Si $\alpha E_{0}^{\omega} \beta$, montrons $f(\alpha)-f(\beta) \in E$. Soient $\varepsilon>0$ et $P$ tels que $\sum_{p \geq P} 2^{-p}<\varepsilon$; dans $f(\alpha)$ et $f(\beta)$ les sommes

$$
a_{p}=\sum_{q} \alpha(p, q) \pi_{\left[n_{\langle p, q\rangle}, n_{\langle p, q\rangle+1}[\right.}\left(x_{p}\right)
$$


et

$$
b_{p}=\sum_{q} \beta(p, q) \pi_{\left[n_{\langle p, q\rangle}, n_{\langle p, q\rangle+1}[\right.}\left(x_{p}\right)
$$

sont identiques à un nombre fini de termes près, et ceci pour tout $p$. Comme chaque terme est à support fini, la différence de ces sommes ne contribue à $f(\alpha)-f(\beta)$ que pour une suite finie. Comme ceci est vrai pour tout $p<P$, on a un nombre $K$ tel que les composantes de $\varepsilon_{K}(f(\alpha)-f(\beta))$ ne proviennent que de différences $a_{p}-b_{p}$ pour des $p \geq P$.

Or ces différences sont des sommes de termes $\pi_{\left[n_{\langle p, q\rangle}, n_{\langle p, q\rangle+1}[\right.}\left(x_{p}\right)$ multipliés par 0,1 ou -1 suivant la valeur de $\alpha(p, q)-\beta(p, q)$.

De toute façon on a $a_{p}-b_{p} \leq_{\omega} x_{p}$ et comme

on a

$$
\varepsilon_{K}(f(\alpha)-f(\beta)) \leq_{\omega} \sum_{p \geq P}\left(a_{p}-b_{p}\right),
$$

$$
\begin{aligned}
\varphi\left(\varepsilon_{K}(f(\alpha)-f(\beta))\right) & \leq \varphi\left(\sum_{p \geq P}\left(a_{p}-b_{p}\right)\right) \leq \sum_{p \geq P} \varphi\left(a_{p}-b_{p}\right) \\
& \leq \sum_{p \geq P} \varphi\left(x_{p}\right) \leq \sum_{p \geq P} 2^{-p}<\varepsilon .
\end{aligned}
$$

On a donc bien $\varphi\left(\varepsilon_{n}(f(\alpha)-f(\beta))\right) \rightarrow 0$ et $f(\alpha)-f(\beta) \in E$.

Si $\alpha E_{0}^{\omega} \beta$, il y a un $p$ tel que $\alpha(p, q) \neq \beta(p, q)$ pour une infinité de $q$, ce qui fait une infinité de $k=\langle p, q\rangle$ tels qu'on ait

$$
\pi_{\left[n_{k}, n_{k+1}[\right.}\left(x_{p}\right) \leq_{\omega} \pi_{\left[n_{k}, n_{k+1}[\right.}(f(\alpha)-f(\beta)),
$$

ce qui prouve que $\varphi\left(\varepsilon_{n}(f(\alpha)-f(\beta))\right) \geq \delta_{p}>0$ pour tout $n$ et donc que $f(\alpha)-f(\beta) \notin E$.

On a donc bien : $\alpha E_{0}^{\omega} \beta \Leftrightarrow f(\alpha)-f(\beta) \in E$.

Une conséquence est que $(f \times f)^{-1}\left(\equiv_{E}\right)$ est $E_{0}^{\omega}$ qui est un vrai $\Pi_{3}^{0}$, et comme $E=g^{-1}\left(\equiv_{E}\right)$ pour l'application continue $g: x \mapsto(0, x), E$ est bien $\Pi_{3}^{0}$-complet.

Corollaire (4.2.3). Si E est un espace héréditaire polonisable analytique dans $\mathbb{R}^{\omega}$, il est fermé, $\mathrm{F}_{\sigma}$-complet ou $\boldsymbol{\Pi}_{3}^{0}$-complet.

Remarque. Comme il a été dit, on peut omettre l'hypothèse que $E$ est analytique (cf. $[\mathrm{C}]$ ).

Preuve. En effet, c'est le résumé des résultats précédents, en notant qu'un tel $E$ peut être un $\mathbb{R}^{A}$, avec $A$ fini ou non, ou isomorphe (s'il n'est pas libre) à un des types d'espaces trouvés.

CONTRE-EXemple (4.2.4). (i) Il y a des espaces héréditaires complètement métrisables de classes boréliennes aussi grandes qu'on veut, et même $(A C)$ il y en a qui ne sont ni analytique ni coanalytique;

(ii) sous $(P D+A C)$, il y en a qui ne sont pas projectifs. 
Preuve. On peut faire correspondre simplement à un idéal libre $\mathcal{I}$ de $\omega$ le sous-espace héréditaire $X$ tel que $c_{0} \subseteq X \subseteq \ell_{\infty}$ :

$$
x \in X \Leftrightarrow x \in \ell_{\infty} \text { et } \forall p>0,\{k|| x(k) \mid>1 / p\} \in \mathcal{I} .
$$

$X$ est alors un fermé de $\ell_{\infty}$ muni de $\|\cdot\|_{\infty}$, donc est un Banach avec cette norme, mais $X \cap 2^{\omega}=\mathcal{I}$, donc la classe descriptive de $X$ est au moins celle de $\mathcal{I}$. Comme on peut aisément trouver des idéaux boréliens de classes arbitrairement grandes, la première assertion est démontrée.

Maintenant, avec (AC), considérons un $\mathcal{I}$ tel que $\mathcal{U}=\{A \mid \omega-A \in \mathcal{I}\}$ soit un ultrafiltre non libre, donc $\mathcal{U}=2^{\omega}-\mathcal{I}$. Alors $\mathcal{U}$ et donc $\mathcal{I}$ ne sont pas Baire-mesurables (on le voit par exemple par l'absurde : s'ils l'étaient, on pourrait appliquer le théorème de Talagrand $[\mathrm{T}]$ qui décompose $\omega$ en $F_{k}$ dont seules les unions finies sont dans $\mathcal{I}$. Mais alors $F_{0} \cup F_{2} \cup F_{4} \cup \ldots$ n'est pas dans $I$ et son complémentaire $F_{1} \cup F_{3} \cup F_{5} \cup \ldots$ non plus, ce qui contredit le fait que $\mathcal{U}$ est un ultrafiltre).

Par conséquent, aucun des deux n'est analytique, donc $X$ ne peut être ni analytique ni coanalytique. Si on a (PD), tout ensemble projectif est Baire-mesurable, donc $\mathcal{I}$ n'est pas projectif, donc $X$ non plus.

\section{Bibliographie}

[C] P. Casevitz, Espaces héréditaires complètement métrisables, Fund. Math., à paraître.

[K] A. Kechris, Classical Descriptive Set Theory, Springer, New York, 1995.

[K-L] A. S. Kechris and A. Louveau, The classification of hypersmooth Borel equivalence relations, J. Amer. Math. Soc. 10 (1997), 215-242.

[K-L-W] A. S. Kechris, A. Louveau and W. H. Woodin, The structure of $\sigma$-ideals of compact sets, Trans. Amer. Math. Soc. 301 (1987), 263-288.

[M] Y. N. Moschovakis, Descriptive Set Theory, North-Holland, Amsterdam, 1980.

[Sc] H. H. Schaefer, Banach Lattices and Positive Operators, Springer, New York, 1974.

[So] S. Solecki, Analytic ideals and their applications, Ann. Pure Appl. Logic 99 (1999), 51-72.

[T] M. Talagrand, Compacts de fonctions mesurables et filtres non mesurables, Studia Math. 67 (1980), 13-43.

SDAD, Université de Caen

Campus II, Boulevard Maréchal Juin

1, Esplanade de la Libération

BP 5186

F-14032 Caen Cedex, France

E-mail: casevitz@math.unicaen.fr 